\title{
OPTIMAL POWER CONTROL FOR NETWORK-CENTRIC AND USER-CENTRIC WIRELESS NETWORKS IN INTERFERENCE-LIMITED FADING CHANNELS
}

by

\section{PIPLU RANI PAUL}

B.Sc. Engg., Bangladesh University of Engineering and Technology, 2002

\begin{abstract}
A THESIS SUBMITTED IN PARTIAL FULFILLMENT OF
THE REQUIREMENTS FOR THE DEGREE OF

MASTER OF APPLIED SCIENCE
\end{abstract}

in

THE FACULTY OF GRADUATE STUDIES

(Electrical and Computer Engineering)

UNIVERSITY OF BRITISH COLUMBIA

August 2005

(C) Piplu Rani Paul, 2005. 


\begin{abstract}
Efficient allocation of transmitter power is of paramount importance in wireless networks for both longer battery life of the mobile devices and increased utilization of the scarce wireless spectrum. Traditional power control scheme updates power whenever the fading state of the channel is changed. This approach consumes a lot of signal processing energy and may be impractical for fast-fading channels. Alternative approach is to take the statistical variation of the signal-to-interference and noise ratio of each transmitter/receiver pair into account and allocate power to optimize outage, power or utility. We address the problem of optimal power control for interferencelimited wireless networks with both Rayleigh faded desired and interference signals assuming latter approach. Unlike most of the works in the literature that use complex non-linear optimization techniques or approximate heuristic- based methods, we propose simple methods to solve the optimal power control problems. We formulate the problems from the viewpoint of both user and network. In outage-based formulations, we minimize the worst outage probability over all transmitter/receiver pairs. In utility-based formulations, which are more suitable for wireless data networks, we consider the problem of maximizing minimum utility over all transmitter/receiver pairs for network-centric scheme and individual utility for user-centric scheme. In all the schemes, we put non-negativity constraints on all the transmitted powers. We also formulate problem that minimizes the total power with specified bounds on the individual outage probability. With appropriate transformation techniques, we convert the complex constrained optimization problems into equivalent unconstrained problems, which are suitably solved using either DFP method or BFGS method. We perform extensive numerical simulations, which reveal that our proposed algorithms are very efficient to converge to the optimal solution with few iterations.
\end{abstract}




\section{Contents}

Abstract

$\begin{array}{ll}\text { Contents } & \text { iii }\end{array}$

List of Tables

List of Figures vii

List of Symbols $\quad$ ix

List of Abbreviations $\quad$ xi

Acknowledgments

Dedication $\quad$ xiii

1 Introduction 1

1.1 Background and Related Works $\ldots \ldots \ldots \ldots$

1.2 Outline of Thesis . . . . . . . . . . . . 10

2 Model Descriptions $\quad 12$

2.1 Introduction . . . . . . . . . . . . . . 12

2.2 Wireless Networks Model . . . . . . . . . . . . . . . 13

2.2 .1 Interference . . . . . . . . . . . . . 15

2.3 Wireless Channel Model $\quad \ldots \ldots \ldots \ldots$ 
2.3.1 Path Loss . . . . . . . . . . . . . . . . . . 16

2.3.2 Multi-path Fading . . . . . . . . . . . . . . . 19

2.4 Wireless System Model . . . . . . . . . . . . . . 20

3 Outage-based Power Control 22

3.1 Introduction . . . . . . . . . . . . . . . 22

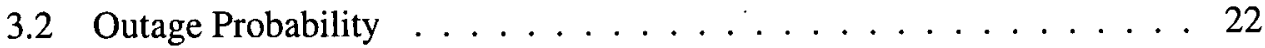

3.3 Outage Based Optimal Power Control . . . . . . . . . . . . 26

3.4 Simulation Results . . . . . . . . . . . . . . . 30

4 Utility-based Power Control 36

4.1 Introduction . . . . . . . . . . . . . . 36

4.2 Utility . . . . . . . . . . . . . . . . . 37

4.3 Utility-based Optimal Power Control . . . . . . . . . . . . . . . 39

$4.3 .1 \quad$ User-centric Case . . . . . . . . . . . . . . . . . . 42

4.3.2 Network-centric Case . . . . . . . . . . . . . . . . 43

4.4 Simulation Results . . . . . . . . . . . . . . . . 46

5 Outage-constraint Total Power Minimization $\quad 62$

5.1 Introduction . . . . . . . . . . . . . . 62

5.2 Problem Formulation . . . . . . . . . . . . . . . 62

5.2 .1 Example: Two Users Case . . . . . . . . . . . . . . . 64

5.3 Numerical Results . . . . . . . . . . . . . . . . . . . 65

6 Conclusions and Future Directions $\quad 68$

6.1 Summary ............................. 68

6.2 Future Works . . . . . . . . . . . . . 70

$\begin{array}{lr}\text { A Appendix } & \mathbf{8 0}\end{array}$

A.1 Proof of Probability Identity $\ldots \ldots \ldots 8$ 
A.2 Davidon-Fletcher-Powell Algorithm . . . . . . . . . . 81

A.3 Broyden-Fletcher-Goldfarb-Shanno Algorithm . . . . . . . . 83 


\section{List of Tables}

3.1 Algorithm for solving outage-based power control to minimize system outage. . . . . . . . . . . . . . . . . . . . . 29

3.2 Data used for simulation in outage-based power control . . . . . . . 30

3.3 Optimal transmitted power vector of system outage probability minimization problem for particular data . . . . . . . . . . 31

4.1 Algorithm for solving utility-based power control in user-centric case . 44

4.2 Algorithm for solving utility-based power control in network-centric case 45

4.3 Data used for simulation in utility-based power control . . . . . . . . 46

4.4 Optimal transmitted power vector of system utility maximization problem for particular data . . . . . . . . . . . . . . . . 47

5.1 Algorithm for solving outage-constraint total power minimization prob-



5.2 Numerical results for example in Section $5.2 .1 \ldots \ldots 6$ 


\section{List of Figures}

2.1 An illustration of wireless cellular network . . . . . . . . . . 13

2.2 Cellular layout: actual coverage shapes and hexagonal footprint approximations ...................... 14

2.3 Variation of path loss, shadowing and multi-path in $\mathrm{dB}$ versus log-distance 17

3.1 Variation of system outage probability with $\mathrm{SINR}^{\text {th }}$ for different mean cross-gain. . . . . . . . . . . . . . . . 33

3.2 Influence of number of transmitter/receiver pairs on system outage probability vs. $\operatorname{SINR}^{t h}$ curve. . . . . . . . . . . . . . . 34

3.3 Dependence of total power on the number of transmitter/receiver pairs . 35

4.1 Conceptual utility for (a) voice and (b) data . . . . . . . . . 38

4.2 Typical variation of individual utility with its transmitted power . . . . 40

4.3 (a) Variation of utility (b) frame success rate with SINR threshold. . . . 50

4.4 Effect of different MPSK ( $M=2,4,8)$ modulation on individual utility function. ...................... 51

4.5 Effect of different MPSK (M=16,32,64) modulation on individual utility function. ........................ 52

4.6 Variation of individual utility function with FSR for different MQAM. . 53

4.7 Dependence of total transmission power on FSR for different MPSK modulation $(\mathrm{M}=2,4,8) . \ldots \ldots \ldots \ldots \ldots$ 
4.8 Dependence of total transmission power on FSR for different MPSK modulation $(\mathrm{M}=16,32,64) . \ldots \ldots \ldots 5$

4.9 Variation of total transmission power on FSR for different MQAM. . . . 56

4.10 Variation of system utility with FSR . . . . . . . . . . . 57

4.11 Variation of system utility with number of $\mathrm{Tx} / \mathrm{Rx}$ pairs. . . . . . . . 58

4.12 Variation of system utility with noise power. . . . . . . . . . . 59

4.13 Variation of total transmitted power with FSR. . . . . . . . . 60

4.14 Variation of total transmitted power with number of $\mathrm{Tx} / \mathrm{Rx}$ pairs. . . . . 61

5.1 Mesh plot of modified objective function in the feasible region . . . . 67

5.2 Contour plot of the modified objective function in the feasible region . 67 


\section{List of symbols}

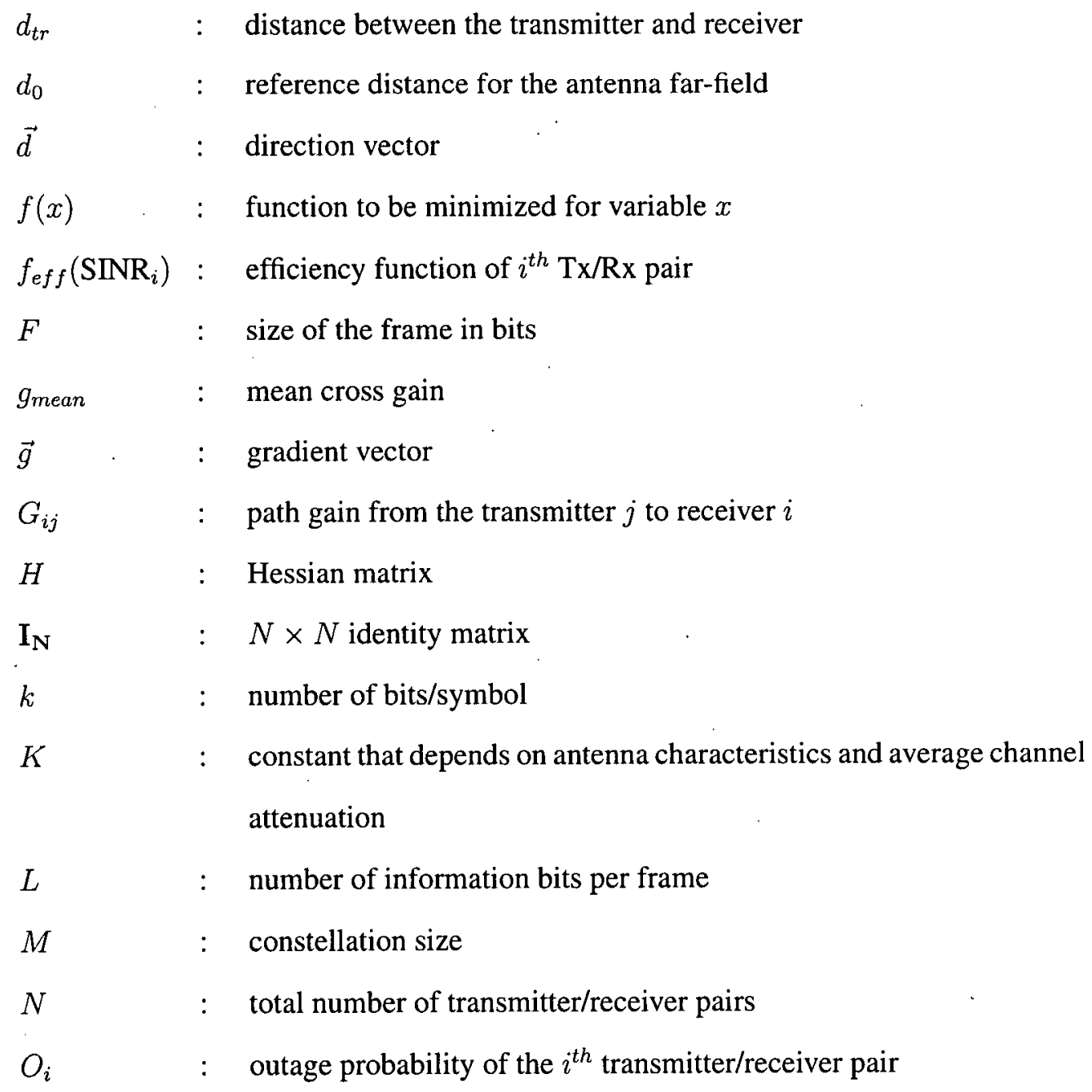




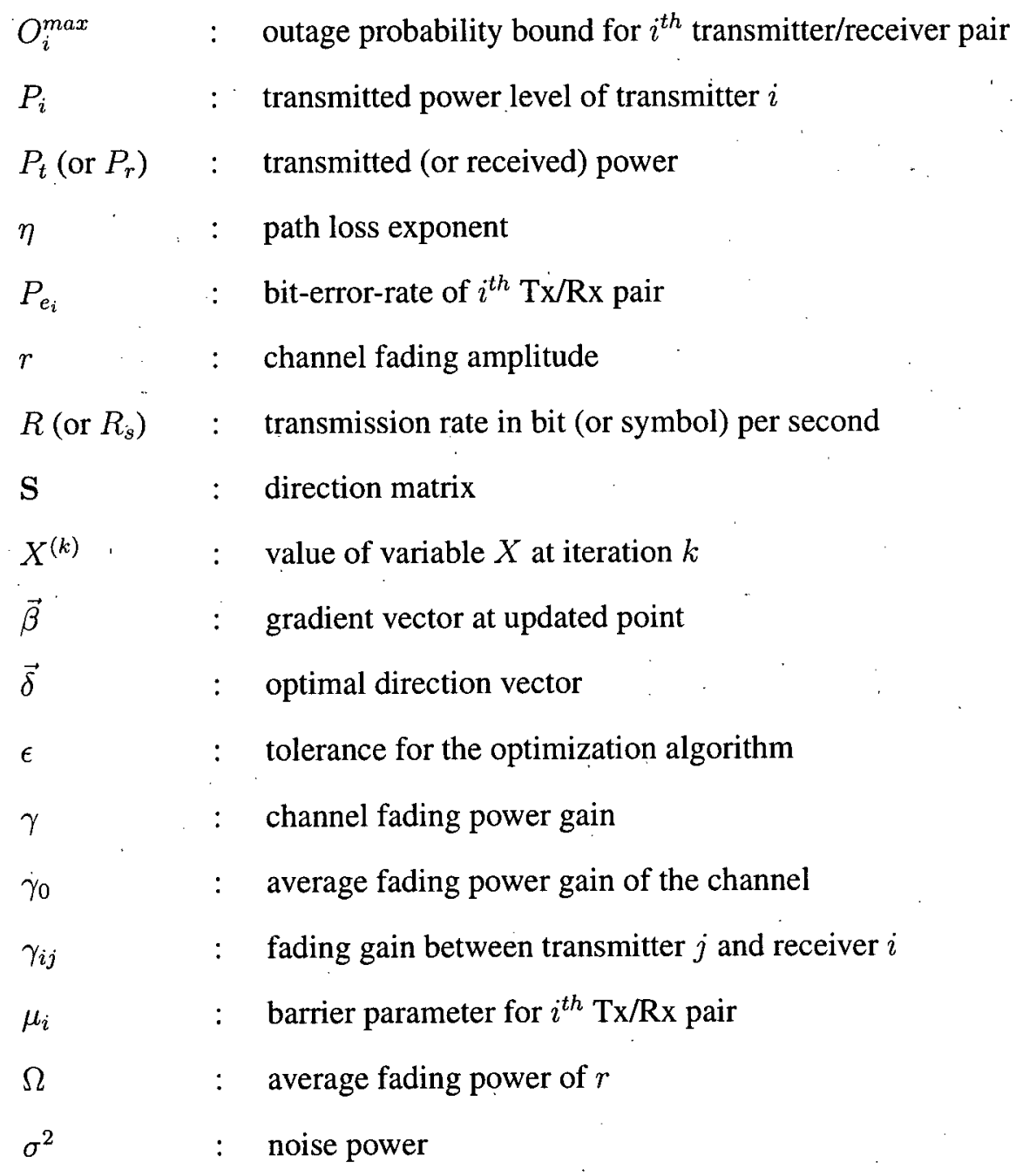




\section{List of abbreviations}

$\begin{array}{lll}\text { AWGN } & : \text { additive white Gaussian noise } \\ \text { BER } & : \text { bit error rate } \\ \text { BFGS } & : \text { Broyden-Fletcher-Goldfarb- Shanno } \\ \text { BS (or MS) } & : \text { base (or mobile) station } \\ \text { CDMA } & : \text { code division multiple access } \\ \text { dB } & : \text { decibel } \\ \text { DFP } & : \text { Davidon-Fletcher-Powell } \\ \text { FDMA } & : \text { frequency division multiple access } \\ \text { FEC } & : \text { forward error correction } \\ \text { FER } & : \text { frame error rate } \\ \text { FSR } & : \text { frame success rate of } i^{\text {th }} \text { Tx/Rx pair } \\ \text { LOS } & : \text { line of sight } \\ \text { MSC } & : \text { mobile switching center } \\ \text { MSE } & : \text { mean square error } \\ \text { PSK } & : \text { phase shift keying } \\ \text { QAM } & : \text { quadrature amplitude modulation } \\ \text { QoS } & : \text { quality of service } \\ \text { RRM } & : \text { radio resource management } \\ \text { TINR } & : \text { signal-to-interference and noise ratio of } i^{\text {th }} \text { transmitter/receiver pair } \\ \text { TDMA } & : \text { transmitter/receiver } \\ & & \end{array}$




\section{Acknowledgments}

During the course of my graduate studies, I have received help and support from many different people, and this thesis would not have been possible without them. First, I would like to express my deepest gratitude and sincere thanks to my thesis advisor, Professor Vijay K. Bhargava, for giving me the opportunity to be a part of his research group and for providing me support and guidance.

I am grateful to all the professors from whom I have learnt a lot during the courses as well as during numerous discussion. I am grateful to Professor Cyril Leung and Dr. Lutze Lampe for their time serving on my M.A.Sc. examination committees. Their suggestions have significantly improved the quality of my thesis.

I am thankful to my former and current group-mates for their friendship, help and cheerfulness. I would also like to thank my friends for adding the element of fun and alleviating the occasional stress during my M.A.Sc. life.

I am indebted to my family for their love and devoted support throughout my life. I would like to acknowledge the commitment and sacrifice of my parents. My brothers have always given me encouragement and support.This thesis as much their as it is mine.

Lastly, but certainly not the least, I would like to express my appreciation to my husband Ashok, for so much. I want to thank Ashok for his patience, unfailing support, and for the many sacrifices he has made over the years. 


\section{Dedication}

To my Family 


\section{Chapter 1}

\section{Introduction}

Wireless communication systems have experienced tremendous growth over the last decade, and this growth continues unabated worldwide. Such developments are mainly driven by strong market demand for personal communication systems and services, which provide ubiquitous and tetherless access to users. The exponential growth of cellular phones, cordless phones, and paging services, coupled with proliferation of laptop and palmtop computers also indicates a bright future for wireless networks [2]. Future wireless networks are envisioned to provide people on the move of the same advanced networking capabilities, such as multimedia applications, Internet access, and guaranteed Quality of Service (QoS), as they enjoy in their homes and or offices. Some even predict that the rapid developments in the field of wireless communication will shrink world into a global communication village by 2010 [3]. To live up to the promise of multimedia communications anywhere and anytime, many technical challenges must be overcome to improve the performance of wireless systems.

The inherent limitations of wireless networks include scarce radio spectrum, highly erratic and essentially stochastic channel (with omnipresent interference, shadowing, and multipath fading), and user mobility. Unlike wired networks, wireless networks with changes in user connectivity and network topology require an integrated and adaptive protocol stack across all layers to achieve acceptable performance [2]. 
Despite all these limitations, the technology and business of cellular communications systems have made spectacular progress since the first systems were introduced 22 years ago and also sparked much of the optimism about the future of multimedia wireless networks [4]. With new mobile satellites, business arrangements, technology, and spectrum allocations make it possible to make and receive telephone calls anytime, anywhere. While first generation $(1 \mathrm{G})$ and second generation $(2 \mathrm{G})$ cellular systems were designed to primarily handle voice traffic, the next wave of wireless systems face new challenges to support high-speed data and multimedia services including facsimile, file transfer, e-mail, and video teleconferencing with different QoS requirements. The global demand for wireless "bandwidth" exhibits, now and in the foreseeable future, significant growth [5]. Compared with wireline networks, wireless resource is very scarce. While more wired network "bandwidth" is created when new physical resources (cable, fiber, router, etc.) are added to the network, wireless communication requires sharing a finite natural resource: the radio frequency spectrum. In wireless communication systems each user transmits its information over the air using some multiple access system. Therefore, effective radio resource management (RRM) is essential to promote the quality and efficiency of a system.

Since the air interface is a shared medium, each user's transmission is a source of interference for others. Further, because of fading, multipath and other impairments, the radio signals get distorted by the time it travels from the transmitter to the receiver. Thus, a common denominator to account for all these impediments is the signal-tointerference and noise ratio (SINR) of the received signals. Furthermore, power consumption is another valuable commodity and users are reluctant to part of it. The satisfaction experienced by someone using a portable device depends on how often the person has to replace or recharge the batteries in the device. Battery life is inversely proportional to the power drain on the batteries. Thus, it is possible to view both SINR and battery energy (transmit power) as commodities that a wireless user desires. There exists a tradeoff relationship between obtaining high SINR and low energy consump- 
tion [6]. Finding a good balance between the two conflicting objectives is the primary focus of the power control component of the RRM.

The 3G Direct-Sequence/Code Division Multiple Access (DS/CDMA) technique is based on spread spectrum communications in which channels are distinguished by special pseudo-noise (PN) sequence. Ideally, each PN sequence is uncorrelated, and each channel is not interfered with others. For detection of the message signal, the receiver needs to know the codeword used by the transmitter. In real situations, there is a near-far problem: the problem of a strong signal masking out a weaker signal, causing unreliable detection of the latter. In addition, the channel power gain for each mobile fluctuates due to multi-path fading and shadowing [7], which gives rise to an additional performance degradation. All of the above limitations make the efficient use of radio resources for high bit rate wireless networks a major challenge. In order to compensate the above influence and interferences, and to increase the system capacity and quality, some modifications are required to make real system approach to theoretical performance. Power control is developed for this purpose.

Power control, the subject of this work, is a fundamental component of the RRM. All modern second-generation $(2 \mathrm{G})$ and third-generation $(3 \mathrm{G})$ radio network interface standards foresee individual power control mechanisms [8] that are carried out by every user terminal and base station (BS) for the entire duration of every call. Power control is intended to provide each user an acceptable connection by eliminating unnecessary interference. It is mainly been used to reduce cochannel interference and to guarantee the SINR of ongoing connections, resulting in a higher utilization and better QoS.

While power control schemes are known to be especially effective and is widely implemented in interference-limited CDMA systems, such as IS-95 [9], it has also been shown to increase the call carrying capacity for channelized systems, such as TDMA/FDMA systems [10]. Furthermore, beyond the conventional concept of power control as a means to eliminating the "near-far" effect, power control plays an important role in interference management, channel quality/service quality provisioning, and ca- 
pacity management [10]- [13]. Thus, power control can be considered a 'control knob' for improving the performance of wireless systems. At the same time, power control enables us to minimize power consumption and hence prolong the battery lifetime in the mobile unit and alleviate health concerns about electromagnetic emission.

QoS refers to the ability of a telecommunications system to provide an appropriate transport service to deliver various types of communications traffic to different users satisfactorily. Sometimes it can be difficult to define the exact technical parameters required to ensure such delivery, especially due to the fact that perception of service quality may differ from one user to another. Thus, it may be observed that whatever global QoS management concept is realized in a network, in a definition it could never produce the same level of satisfaction to each and every user. This becomes even more complicated in cellular network where the interface between the network and users is realized via radio connection and that is not stationary. The non-stationarity of the connectivity in cellular networks is not only due to some of the circumstances common to any kind of telecommunications, but also due to the inherent mobility features of cellular networks: the unexpected and ever changing physical location of mobile users. It is obvious that if a user terminal is located within an optimal distance and in favorable radio visibility conditions, it would greatly increase chances of successful communication with high QoS. On the contrary, being located near the edge of the coverage area (cell) makes communication more difficult and resource demanding [14].

In mobile telephony, the QoS, i.e., the possibility to guarantee the most suitable service level to each different traffic category, is often expressed in terms of outage probability. In this case, the target is to achieve the minimum acceptable SINR. The QoS objective for data differs from the QoS objective for telephones. For data, QoS specifications include frame error rate (FER), frame success rate (FSR), etc. The reason behind this is the contrast between the voice and data traffic. While voice traffic is delay intolerant, error tolerant, and periodic; data traffic is typically delay tolerant, error intolerant, and bursty. 
Adaptive controls of transmitting power at the user terminal (mobile station) as well as at the BS are implemented to optimize the performance of a given communication link by properly balancing the radio link budget. Keeping the transmitter power to the least sufficient levels allows conserving cell capacity (in CDMA systems) and equalizing received signals, while also minimizing co-channel interference to other cells of the system or other systems.

\subsection{Background and Related Works}

Power control has received significant interest from both academic and industrial researchers. Various power control algorithms have been proposed in the literature [10],[15][47]. The elegant works of Yates [15] abstracts the important properties of various power control algorithms and presents a unified treatment of power control. Existing power control algorithms can be categorized into two classes: signal-based and SINR based power control. Signal based power control [16],[17] adjusts the transmission power based on the received signal strength. In contrast, SINR-based power control $[10],[18]-[20]$ changes the power according to the ratio of signal and interference plus noise power levels. Power control based on the ratio of signal power to interferenceplus-noise power is more realistic and is actually desirable from the radio link performance perspective, since it is SINR that determines the received bit error probability [21].

In [22]-[25], fast fading of the joint interference has been modeled by a Rayleigh distribution. French in [26] considered the problem of cochannel interference for Rayleigh fading and log-normal shadowing with one interferer present at any time. Prasad and Arnbak [25] describes combined shadowing and Rayleigh fading of one individual signal as a log-normal distribution with increased variance, and a mean decreased by 1.5 decibel (dB). In [27], Aein investigates cochannel interference management in satellite systems. The author introduces concept of SIR balancing, which yields a "fair" dis- 
tribution of the interference in the sense that all users experience the same SIR level. The problem is identified as an eigenvalue problem for positive matrices. In [28]- [29], Nettleton and Alavi have applied and extended these results to spread spectrum cellular radio systems. In these systems, the adjacent channel interference also has taken into account. They have shown that SIR balancing substantially improves the capacity of such systems.

Power control schemes can be broadly either centralized or distributed. Various centralized and distributed schemes have been proposed in the literature. Centralized and distributed transmitter power control schemes that minimize the outage probability due to co-channel interference in cellular radio systems have been investigated in $[10],[20],[30]$. In [10] the authors have studied performance bounds for power control algorithms. The structure of the optimum algorithm shows that efficient power control and dynamic channel assignment algorithms are closely related. In [31] the authors have proposed a centralized power control scheme considering adjacent-channel interference. They have also shown that a distributed discrete power control algorithm can approximate the behavior of the optimized centralized algorithm by using only limited path gain information. In [32], the performance in a mobile radio system has been investigated in the presence of Ricean fading, log-normal shadowing and deterministic dual path loss, and the possible use of diversity reception and power, for both the hexagonal and linear cell layouts. They have shown the effect of propagation parameter on outage probability. Centralized and distributed algorithms proposed in [33] have been found to update the powers of the mobiles whenever the channel characteristics change, assuming that the rate of change of channel is fairly slow and allowing the change to be tracked accurately. Convergence of these algorithms has been proved for both synchronous and asynchronous CDMA applications. In the case where channel link gains are not exactly known but are estimated only, [34] has proposed a distributed power control algorithm based on interference measurements at the receiver which is proved to converge in the mean-square sense using stochastic approximation based ideas. One 
of the most well-known distributed algorithms originally proposed in [18] has been further studied in several papers, including [35] and [36]. This algorithm is distributed and autonomous because it relies only on local information.

Since outage is an important consideration in the design of cellular and mobile systems, it has been the subject of a number of recent investigations. Cox [37], Yeh and Schwartz [38] have considered outage probabilities due to multiple log-normal interferers. Cox [37] have evaluated the sum of identically distributed interference powers by means of a Monte Carlo study, and computed the outage probabilities for a fixed mobile located in the corner of either a hexagonal or a square cell. The result shows that when the systems are considered on the basis of SINR at the corners of the coverage area, the hexagonal structure is better than squares. In [38], the authors have analyzed arbitrary combinations of interferers for a host of system parameters. The authors have shown that the outage probabilities for both the mobile-to-base and baseto-mobile do not differ in a significant way. The probability distribution functions are approximated by matching means and variances. Muanmmar and Gupta [23] have used a model that took the six closest surrounding interferers into consideration and approximated the distribution of the sum of their amplitudes by a normal distribution for the case of Rayleigh fading. A log-normal distribution approximation has been used for the case of both Rayleigh fading and log-normal shadowing. Chan [39] has derived expressions for co-channel interference probabilities in the presence of multiple, equal averaged-power interferers with Rayleigh fading and log-normal shadowing. Sowerby and Williamson in [40] have considered the problem of outage in the presence of multiple Rayleigh interferers with log-normal shadowing. Linnartz [41] express the outage probability in the presence of log-normal and Rayleigh fading avoiding approximation of the probability density function (pdf) of the received power of various signals. Closed form expressions for outage probabilities of mobile radio channels experiencing multiple, cochannel, independent Nakagami interferers are derived in [33]. In [42], outage probability equations have been used to determine the quality of radio reception 
when that quality is limited by a minimum required signal level, interference from a cochannel transmitter, and variability in the received signal levels. Considering Rayleigh fading and lognormal shadowing as joint causes of signal variation, average outage probability expressions are derived. These expressions have been used to investigate the influence of various system and propagation characteristics on reception quality. It has been shown that the characteristics of radio propagation affect the average quality of reception within an area bounded by a particular outage probability contour. In [47], the authors have provided a simple approximation to the outage probability of the uplink for a CDMA system utilizing an antenna array for the purpose of space diversity. They take stochastic models of the power control error, small-scale fading, and voice activity into account.

In [48], the authors have proposed a power control scheme for Rayleigh/Rayleigh fading environments in which the power need not to be updated whenever the channel meandering from one fading state to another. They have formulated the problem as a complex geometric program and give a heuristic based Perron-Frobenius eigenvalue method to solve the power control problems approximately: In [49], an optimal power control problem. with outage probability specifications is solved using a iterative technique for Rayleigh fading case. The authors also combines multiuser detection techniques with the power control algorithm.

The schemes discussed so far are mainly for cellular telephony systems. Cellular telephony systems only provide circuit-switched voice service with relatively low requirements on bandwidth, bit error rate (BER), and spectral efficiency. Since future wireless systems are to support multimedia applications over wireless fading channels, it is natural to look for a new power control algorithms that are more appropriate for multimedia wireless systems. Many of wireless multimedia services are in the category of wireless data communications. The key for meeting the increasing demand for wireless data communications is the development of high-performance radio systems, which take the unique features of data service into account. Unlike voice traffic, data 
traffic trends to be highly bursty and tolerates much lower transmission errors, but has less stringent delay requirements. As a result, techniques like packet switching, retransmission and forward error correction (FEC), and link adaption are necessary for data applications. Correspondingly, the power control problem for wireless data has to be formulated differently. The power control problem for systems of single-class wireless data is formulated in [51] using concepts from microeconomics and game theory.

In [13],[50],[52], a power control problem for data communications is formulated as a non-cooperative $\mathrm{N}$-person game in which each mobile transmits a power level that maximizes user's utility. They have shown their algorithm converges to Nash equilibria. Further, in [13], [52], [81] the authors have shown that, by introducing pricing, system efficiency can be improved. In these works, the BS informs each mobile of a fixed price per unit power and each mobile transmits at a power level that maximizes its net utility (utility minus cost for power allocation). They have shown that the system utilization significantly depends on the choice of price. In [53],[54] a downlink resource allocation problem is considered with restricted types of utility functions. In [53], only voice services are considered and utility functions are modeled as step functions, and in [54], utility functions for data are modeled as concave functions. In these works authors obtain the optimal prices for maximizing the total system utility and the total revenue. In [55], heterogeneous sources are considered, where each user is characterized by on-off transmissions that occur on a fast time-scale. The objective of power control is to satisfy the SINR requirement in a statistical sense. To take into account the random, bursty natures of the interferers, the proposed power control algorithm includes a measurement of the variance of the interference. By observing temporal correlation of co-channel interference in broad-band, packet-switched TDMA systems, Leung proposed a Kalman filter method for power control in [56]. Power-included multiple-access schemes are proposed in [57]-[59] based on analytical results obtained for a single link operating in a channel with random interference by minimizing the sum of power cost and backlog cost. Using these multiple-access schemes, power increases in interference first to re- 
duce the backlog; it decreases in interference after a turning point and before a turnoff point is reached; and then it remains zero. The turning point and turnoff point depend on the number of backlogs and the interference distribution. In [60], [61] capacity regions, and optimal power and rate allocation schemes are studied from an information theoretic point of view.

\subsection{Outline of Thesis}

In this thesis, we address the issue of optimal power control algorithms for interferencelimited Rayleigh/Rayleigh fading environments. We give algorithms for both the usercentric and network-centric case. While traditional power control algorithms update the power level when the channel transit from one fading state to another, our algorithm updates power level with statistical variations of SINR to minimize fading-induced outage. That is, power level updates take place at a time scale far larger than Rayleigh fading time scale, which is often the log-normal time scale. This approach is more practical for fast-fading channel and saves signal processing energy. In previous literature, the authors either take complex non-linear optimization-based method to find the optimal solutions or heuristic-based method employing Perron-Frobenius eigenvalue theory to find approximate solutions. In contrast, we present simple and equivalent unconstrained optimization-based formulation and give the algorithms that are very efficient to find optimal power. The outline of the thesis is given below.

In Chapter 2, we describe various models for our study. In particular, we describe the general wireless cellular network model, the characteristics of wireless channels on which they operate, the system model to be used in this work. We define the SINR in this chapter, which is the base of all of our algorithms.

Outage probability is a measure of the quality of the radio reception at a given location in a cellular mobile system with interferences. The QoS of a particular user can be specified in terms of outage probability. In Chapter 3 , we address the problem 
of optimal power control for interference-limited wireless network in which QoS requirements for users are provided in terms of outage. We provide the formulation and solution techniques of the outage-based optimal power control from network-centric point of view. Some simulation results are given to show efficiency and applicability of the proposed algorithm.

In Chapter 4, we formulate the power control problem for data in a interferencelimited wireless network adopting the vocabulary and mathematics of microeconomics in which the QoS objective is referred to as a utility function. The utility function of data signals is different from the telephone utility function [51]. While the minimum SINR constraint-based allocation of resources is appropriate for voice, it does not represent data user satisfaction well. The perception of voice quality remains unchanged beyond a certain SINR. However, data services are sensitive to changes in the SINR in a continuous fashion. We provide formulation and solution technique for optimal power control for both the user-centric and the network-centric schemes with the goal of optimizing utility under constraints on transmission powers of all transmitter/receiver pairs. At last, we give simulation results to portrait the convergence of the algorithms, and variations of utility and power with different parameters.

While in Chapter 3 our goal is to minimize fading-induced outage with constraints on all transmitter's powers, in Chapter 5 the objective is to minimize the total transmitting powers keeping the outage below some specified bounds. We formulate the problems first as a constrained problem, then we give the equivalent unconstrained formulation that is computationally easier and efficient to solve. We discuss and simulate a simple example to show the efficiency of convergence of the algorithms.

We summarize our contributions in the thesis and indicate some future research in Chapter 6. The probability identity used to derive the expression for outage, detailed Davidon-Fletcher-Powell (DFP) and Broyden-Fletcher-Goldfarb-Shanno (BFGS) algorithms are given in Appendix A.1, Appendix A.2 and Appendix A.3 respectively. 


\section{Chapter 2}

\section{Model Descriptions}

\subsection{Introduction}

Cellular mobile radio systems are designed to provide services to thousands of users in a very limited bandwidth. Good quality service, efficient spectrum utilization, and cost effectiveness are the fundamental aims of modern mobile radio system design. Cellular radio systems attempt to provide a telephone service to the mobile public with a quality as good as that afforded by conventional landline services. However, the deliberate reuse of radio channels over relatively short distances in cellular radio means that reception quality can be limited by interference.

Cellular radio systems have evolved significantly in the past two decades of their existence. While first generation $(1 \mathrm{G})$ and second generation $(2 \mathrm{G})$ cellular systems were designed to primarily handle voice traffic, third generation (3G) systems offer significant data traffic handling capabilities. The research community is now working on the fourth generation systems, which promise to be significantly more sophisticated and support high data rate multimedia traffic.

In this chapter, we describe the models for our work. We discuss the general wireless cellular model in Section 2.2. The cellular systems are limited by interference. The sources of interference is discussed in Section 2.2. The channel model and the system 


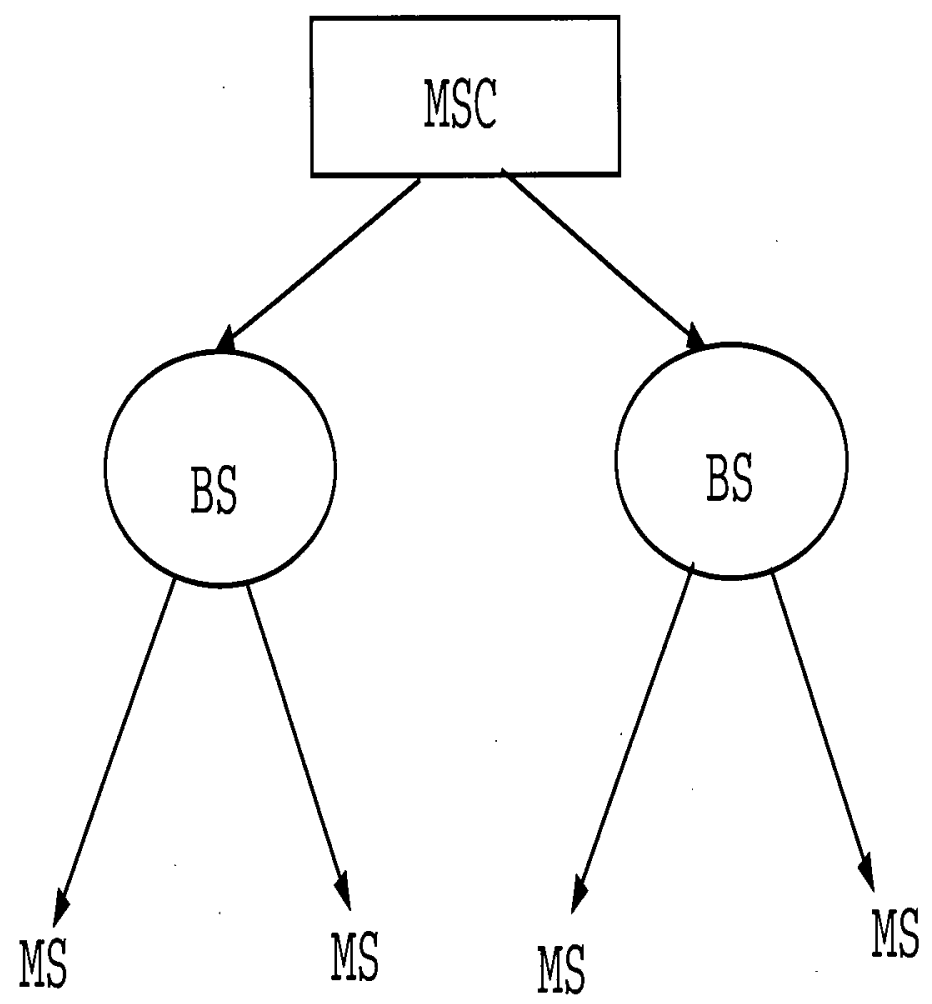

Figure 2.1: An illustration of wireless cellular network.

model of our work are given in Section 2.3 and Section 2.4 respectively.

\subsection{Wireless Networks Model}

A wireless communication network offers a flexible information transport platform that allows mobile users to roam without suffering intolerable performance degradation. A wireless networking scenario is depicted in Fig. 2.1. The geographical area that a particular company covered is divided into a number of cells. A cell is served by a BS supporting the services of many mobile stations. In other words, a BS is the information distribution center for all mobile stations (MSs) within its signalling coverage area. The signal propagation medium between the MSs and the BS is wireless. Several cells constitute a cluster (e.g., 7) and the available channels are replicated in each cluster. 
This is called frequency reuse. The radio channel from a mobile station to its serving BS is called the uplink or reverse channel, and the radio channel from the BS to the MSs is called the downlink or forward channel. Base stations are connected to Mobile Switching Centers (MSCs) by wirelines to extend the geographical coverage of a single BS. An MSC may be connected by wirelines to other MSCs and/or to a wired backbone network. The actual coverage area of a cell, over which a minimum QoS (in terms of

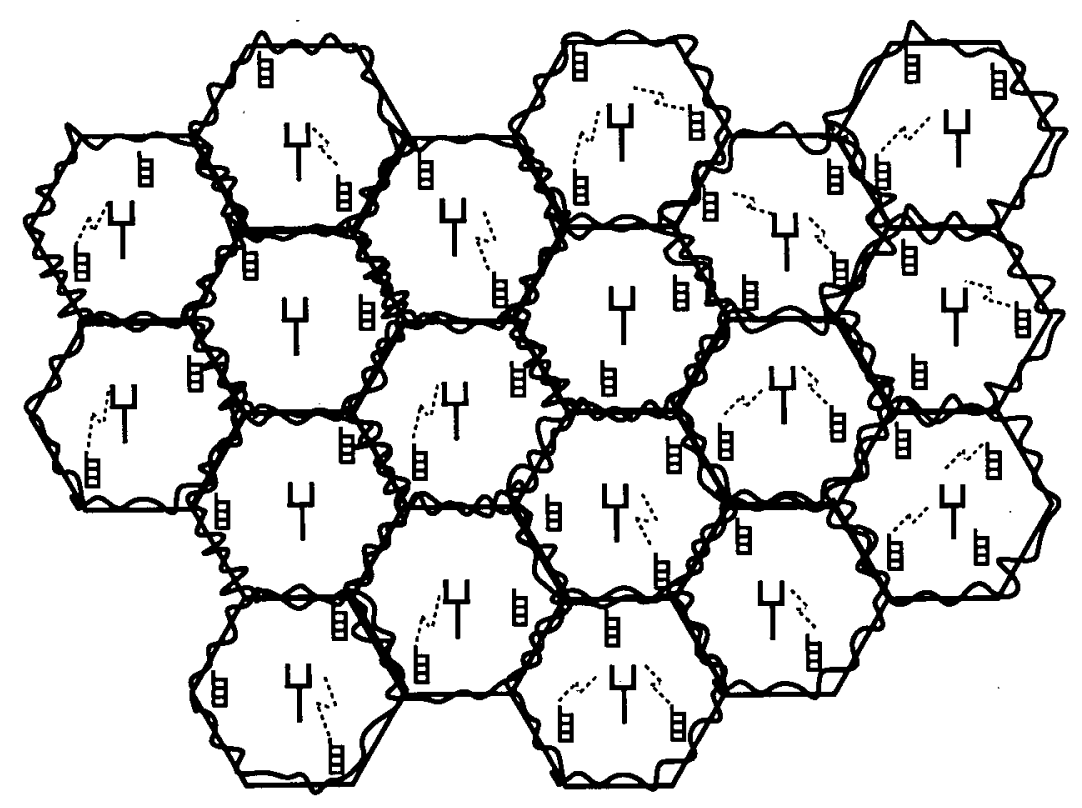

Figure 2.2: Cellular layout: actual coverage shapes and hexagonal footprint approximations

SINR, for example) can be guaranteed, is known as its footprint [7]. The footprint's shape depends on propagation conditions existing in the cell [62]. Hexagonal, circular, and square footprints are typical footprint shape approximations used in the literature analyzing cellular systems. This is because of the well known fact that regular hexagons 
and squares tessellate, i.e., cover a two-dimensional plane without overlapping. The shapes in real cellular system deployments may vary from these approximations due to shadowing, fading, etc. For example, a study [62] based on power measurements conducted at $900 \mathrm{MHz}$ in Manhattan indicates that footprint is a concave diamond. A typical cellular layout with a hexagonal footprint is shown in Fig. 2.2. Also shown in the figure are the constant SINR lines that determine the actual cell shape.

\subsubsection{Interference}

Interference is the major limiting factor in the performance of wireless communication networks. Source of interference include another mobile in the same cell, other BSs operating in the same frequency band, or any noncellular system which inadvertently leaks energy into the mobile frequency band. Interference is more sensitive in urban areas, due to the greater RF noise floor and the large number of base stations and mobiles. Interference is more significant than the thermal noise. While thermal noise can be overcome by increasing the transmit power, doing so does not help in interferencelimited systems since the interference power proportionally increases [63].

Two major types of system generated interference are [7]

- Co-Channel Interference: In a given coverage area there are several cells that use the same set of frequencies, these cells are called co-channel cells. The interference between signals from co-channel cells is called co-channel interference.

- Adjacent Channel Interference: Interference resulting from signals which are adjacent in frequency to the desired signal is called adjacent channel interference. Adjacent channel interference results from imperfect receiver filters which allow nearby frequencies to leak into the passband. The problem can be particularly serious if an adjacent channel user is transmitting in very close range to a subscriber's receiver, while the receiver attempts to receive a BS on the desired channel. This is referred to as the near-far effect. 


\subsection{Wireless Channel Model}

The wireless radio channel poses a severe challenge as a medium for reliable high-speed communication. It is not only susceptible to noise, interference and other channel impediments, but also these impediments change over time in unpredictable ways due to user movement. When electromagnetic waves travel through the environment, they are reflected, scattered, and diffracted by walls, buildings, natural terrain, and other objects. Characterizing this propagation in detail calls for solving Maxwell's equations with boundary conditions determined by the physical characteristics of the obstructing objects [64]. These characterizations are very complex, and often physical characteristics are themselves unknown. However, considerable efforts have been devoted to the statistical modeling and characterization of these different effects. The result is a range of relatively simple and accurate statistical models for wireless channels which depend on the particular propagation environment and the underlying communication scenario.

\subsubsection{Path Loss}

Propagation models that predict the mean signal strength for an arbitrary transmitterreceiver (Tx-Rx) separation distance are useful in estimating the radio coverage area of transmitter and are called large-scale propagation models since they characterize signal strength over large Tx-Rx separation (several hundreds to thousands of meters). By using path loss models to estimate the received signal level as a function of distance, it becomes possible to predict the SNR for a mobile communication system. But the complexity of signal propagation makes it difficult to obtain a single model that characterizes the path loss accurately across a range of different environments. However, for general tradeoff analysis of various systems designs it is sometimes best to use a simple model that captures the essence of signal propagation without resorting the complicated path loss models.

- Log-distance path loss model: As a mobile user moves away from its BS, the 


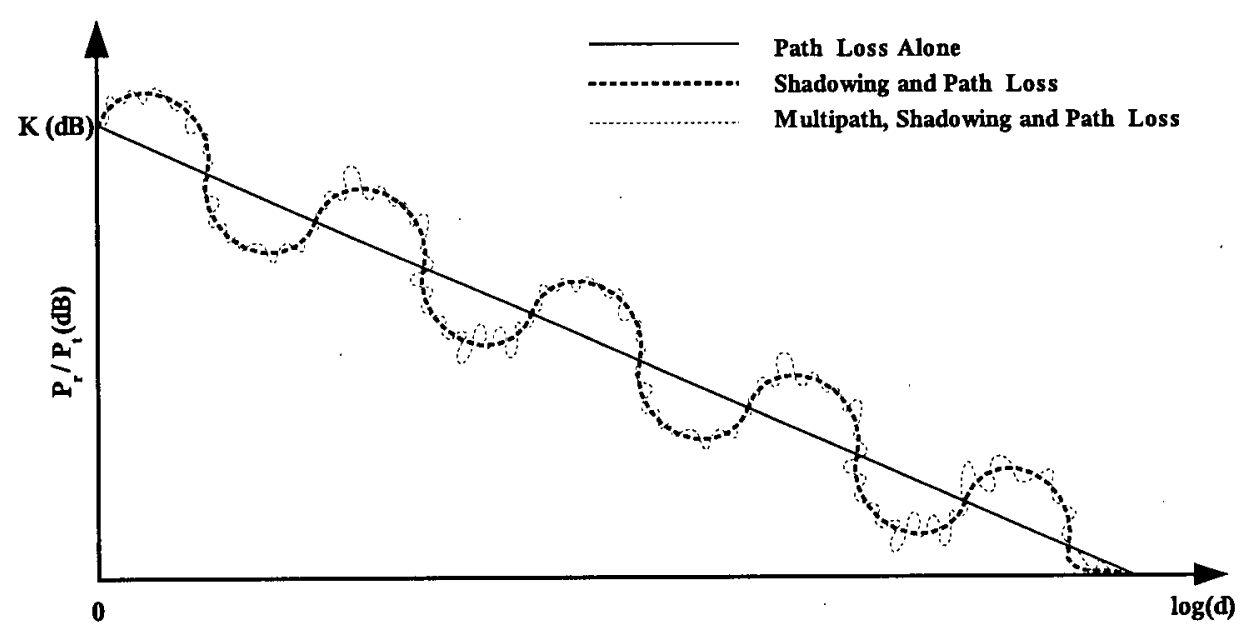

Figure 2.3: Variation of path loss, shadowing and multi-path in $\mathrm{dB}$ versus log-distance received signal becomes weaker because of the growing propagation attenuation with distance. Both theoretical and measurement-based propagation models indicate that average signal power decreases logarithmically with distance, whether in outdoor or indoor radio channels. Thus, the following simplified model for path loss as a function of distance is commonly used for system design:

$$
P_{r}=P_{t} K\left[\frac{d_{0}}{d_{t r}}\right]^{\eta}
$$

The received power in $\mathrm{dB}$ is thus

$$
\dot{P_{r}}(\mathrm{dBm})=P_{t}(\mathrm{dBm})+K(\mathrm{~dB})-10 \eta \log _{10}\left(\frac{d_{t r}}{d_{0}}\right)
$$

The pathloss is the difference (in $\mathrm{dB}$ ) between the effective transmitted power and received power and thus:

$$
P L(\mathrm{~dB})=-K(\mathrm{~dB})+10 \eta \log _{10}\left(\frac{d_{t r}}{d_{0}}\right)
$$

In this approximation, $K$ is a unitless constant which depends on the antenna characteristics and the average channel attenuation, $d_{0}$ is a reference distance for 
the antenna far-field, and $\eta$ is the path loss exponent. Due to scattering phenomena in the antenna near-field, the model (2.1) is generally only valid at transmission distances $d_{t r} \geq d_{0}$, where $d_{0}$ is typically assumed to be $1-10 \mathrm{~m}$ indoors and $10-100 \mathrm{~m}$ outdoors. The value $K<1$ is sometimes set to the free space path loss at distance $d_{0}$ :

$$
K(d B)=-20 \log _{10}\left(\frac{4 \pi d_{0}}{\lambda}\right)
$$

Alternatively, $K$ can be determined by measurement at $d_{0}$ or (alone or together with $\eta$ ) by minimizing the mean square error (MSE) between the model and empirical measurements [65]. The value of $\eta$ depends on the propagation environment. For example, in free space $\eta$ is equal 2, and when obstructions are present, $\eta$ will have a larger value (e.g., in urban area $\eta$ varies from 2.7 to 5)

- Log-normal shadowing: Furthermore, as the mobile moves in uneven terrain, it often travels into a propagation shadow behind a building or a hill or other obstacle much larger than the wavelength of the transmitted signal, and the associated received signal level is attenuated significantly. This phenomenon is called shadowing. Since the location, size and dielectrics properties of the blocking objects as well as the changes in reflecting surfaces and scattering objects that causes the random attenuation are generally unknown, statistical models are widely used to characterize this attenuation. A log-normal distribution is a popular model for characterizing the shadowing process. That is, log-normal shadowing describes the random shadowing effects which occur over a large number measurement locations that have the same Tx-Rx separation, but have different levels of clutter on the propagation path. In simple, the log-normal shadowing implies that measured signal level at a specific $\mathrm{Tx}-\mathrm{Rx}$ separation have a Gaussian (normal) distribution given by:

$$
p\left(\psi_{d B}\right)=\frac{1}{\sqrt{2 \pi} \sigma_{\psi_{d B}}} \exp \left(-\frac{\left(\psi_{d B}-\mu_{d B}\right)^{2}}{2 \sigma_{d B}{ }^{2}}\right)
$$

where $\mu_{d B}$ is the mean and $\sigma_{\psi_{d B}}$ is the standard deviation in $\mathrm{dB}$. 
- Combined path loss and shadowing: Model for path loss and shadowing are typically superimposed to capture power falloff versus distance along with the random attenuation about this path loss from shadowing. For this combined model the path loss in $\mathrm{dB}$ is given by:

$$
P L(\mathrm{~dB})=-K(\mathrm{~dB})+10 \eta \log _{10} \frac{d_{t r}}{d_{0}}+\psi_{d B}
$$

where $\psi_{d B}$ is a Gaussian-distributed random variable with zero mean and variance $\sigma_{d B}^{2}$.

\subsubsection{Multi-path Fading}

Small scale or multipath fading is the rapid fluctuation of the amplitude of a radio signal over a short period of time or travel distance. Multipath fading is caused by interference between two or more versions of the transmitted signal which arrive at the receiver at slightly different times. These multipath signals combine at the receiver antenna to give a resultant signal which can vary widely in amplitude and phase, depending on the distribution of the intensity and relative propagation time of the waves and the bandwidth of the transmitted signal. Propagation models that characterize the rapid fluctuations of the received signal strength over very short travel distances (a few wavelengths) or short time durations (on the order of seconds) are called small scale or fading models. We shall discuss the Rayleigh fading model that is frequently used to model multipath fading with no direct line-of-sight (LOS) path between the transmitter and receiver antennas. This model applies to macrocellular radio mobile systems as well as to tropospheric, ionospheric, and maritime ship-to-ship communication. 


\section{Rayleigh Fading Model}

The Rayleigh distribution has a pdf given by [7],

$$
p(r)= \begin{cases}\frac{2 r}{\Omega} \exp \left(-\frac{r^{2}}{\Omega}\right), & \text { if } 0 \leq r \leq \infty \\ 0, & \text { if } r<0\end{cases}
$$

where $r$ is the channel fading amplitude, and $\Omega=\overline{r^{2}}$ is the average fading power of $r$. The instantaneous fading power gain of the channel $\gamma$ is distributed according to an exponential distribution given by [66],

$$
p_{\gamma}(\gamma)=\frac{1}{\gamma_{0}} \exp \left(-\frac{\gamma}{\gamma_{0}}\right)
$$

where $\gamma_{0}=\mathbb{E}\{\gamma\}$ is the average fading power gain of the channel and $\mathbb{E}\{$.$\} is the$ expectation operator.

\subsection{Wireless System Model}

We consider a cellular wireless system that consists of fixed $N$ number of channels (e.g., frequency channels, codes in a CDMA systems, or antenna beams in an antenna array). One channel is assigned to a particular transmitter/reciever pair at a particular time. Suppose $P_{i}$ denote the transmit power level of transmitter $i$. The receiver associated with transmitter $i$ is denoted as receiver $i$. By transmitter and receiver, we do not necessarily mean different physical transmitters and receivers; different receivers, for example, might refer to the same physical receiver with different frequency channels, codes, or antenna beams in an antenna array. Let $G_{i j}$ is a positive number and denotes the path gain from the transmitter $j$ to the receiver $i$. It can represent distance dependent power attenuation, log-normal shadowing, cross-correlations between codes in a CDMA systems, as well as gain dependency on antenna direction.

We assume Rayleigh/Rayleigh fading environment in which both desired signal and interference signals are subject to Rayleigh fading. This assumption of Rayleigh/Rayleigh 
fading environment is very realistic in urban wireless networking environments since the receiver gets no direct line-of-sight signal components, either from its own transmitter or from the interfering transmitters. The received signal amplitude in a Rayleigh fading environment has a Rayleigh distribution given by (2.7) and the channel fading power gain, which is proportional to the square of the signal amplitude, is exponentially distributed and given by (2.8).

Let $\gamma_{i j}$ denote the fading gain between transmitter $j$ and receiver $i$. Note that whereas gain $\gamma_{i j}$ is randomly time-varying, gain $G_{i j}$ does not change much with time. We assume that $G_{i j}$ are constant and hence the analysis holds for a time scale over which the factors that determine $G_{i j}$ are approximately constant. Therefore, the power received at receiver $i$ from transmitter $j$ is an exponentially distributed random variable

$$
\gamma_{i j} G_{i j} P_{j}
$$

with expected value

$$
\mathbb{E}\left[\gamma_{i j} G_{i j} P_{j}\right]=G_{i j} P_{j}
$$

where the mean value of fading gain, $\mathbb{E}\left[\gamma_{i j}\right]=\gamma_{0}$ is unity.

The instantaneous SINR of the receiver $i$ is given by,

$$
\operatorname{SINR}_{i}=\frac{\gamma_{i i} G_{i i} P_{i}}{\sum_{k \neq i} \gamma_{i k} G_{i k} P_{k}+\sigma^{2}},
$$

where, the term in the numerator denotes the desired received power of the receiver $i$ from transmitter $i$ and the denominator is due to the total interference due to received power from all other transmitters and the noise power due to the white channel noise and thermal noise at the receiver. 


\section{Chapter 3}

\section{Outage-based Power Control}

\subsection{Introduction}

Outage probability is an important measure in the design of cellular mobile systems to operate in a fading environment with multiple users. In the previous chapter, we have presented the system model and channel model for our work. An unconstrained optimization based power control algorithm for the interference-limited Rayleigh fading wireless networks is given in this chapter. The QoS requirements is achieved in terms of outage probability. We discuss the optimal algorithm for the network-centric case [1]. In section 3.2, we derive the expression for outage probability and its equivalent expression suitable for optimization. The formulation and solution techniques for minimizing system outage are described in Section 3.3. Simulation results are given in Section 3.4 .

\subsection{Outage Probability}

"Outage probability" is a statistical measure that describes the probability of failing to achieve "adequate reception" of a signal over the intended area [42]. The term outage is closely related to the criterion used for the assessment of the satisfactory reception [67]. 
Two criteria are in common use. In the first criterion, the reception is considered to be satisfactory if the desired signal power exceeds the sum of interference and noise powers. In the second criterion, the satisfactory reception needs, in addition to the above criterion, to meet a requirement of a minimum signal power. Therefore, to achieve "adequate reception", the short-term desired signal must be simultaneously greater than both the some minimum signal level and the short-term sum of the interfering signals and noise powers by a margin known as the interference protection ratio. The protection ratio serves as a means of quantifying the vulnerability of reception quality to co-channel interference. Outage occurs when the above criteria are not satisfied. Due to limited spectrum availability, radio networks become more and more limited by mutual interference between users. Therefore, outage probability is usually determined in terms of interference between different user's frequency channels, or codes.

Outage probability can be expressed mathematically as [68],

$$
O=1-\int_{\mathrm{sig}_{\min }}^{\infty} p(\mathrm{sig}) \times \int_{0}^{\mathrm{sig} / r_{p}} p(\text { int }) \times d(\text { sig }) \times d(\text { int })
$$

where,

$p($ sig $)=$ the pdf of the desired signal power,

$p($ int $)=$ the pdf of the resultant interfering signal power,

$r_{p}=$ the signal-to-interference protection ratio and

$\operatorname{sig}_{\min }=$ the minimum required signal power for maintaining connection.

The outage probability of a cellular mobile radio system depends on the channel characteristics in which it operates. Therefore, in order to calculate the outage probability at a given location, it is necessary to know the pdf of the desired and interfering signals. The outage probability for different propagation medium has been derived in different literature (as for example [41], [33], [39], [42], etc). Traditionally, both the centralized and distributed power control schemes allocate power assuming that the wireless link gains are quasi-stationary or slowly-varying. These schemes base their power-control on the observed signal-to-interference and noise ratio at the receiver or 
on the knowledge of the gains of all the links. Therefore, the power control algorithms update the allocated power whenever the fading state of the channel changes or the gain of any link changes. These approaches of power control has two limitations: firstly, tracking fast variations of fast fading channels is very difficult and may not always be practical. Secondly, very frequent updates consume a lot of signal processing energy. A power control scheme in which the power does not need to be updated whenever the channel meanders from one fading state to another is proposed in [48]. The statistical variation of the SINR of each $\mathrm{Tx} / \mathrm{Rx}$ pair is taken into account and power updates are carried out at a time scale far larger than the Rayleigh fading time scale. Between successive power-control updates, outage occurs because of fast fading (of both signal and interference) and log-normal shadowing, and distance dependent attenuation remain constant. Clearly, the probability of outage can be reduced by allocating power in such a way that each mobile has an extra margin of SINR, i.e., its SINR is somewhat above the minimum $\operatorname{SINR}^{\text {th }}$ value required for reception. Increasing the margin of SINR reduces the probability of outage, but costs extra power.

In our work, we adopted the latter approach ás [48]. In [48], the optimal power control problem is formulated as a complex nonlinear convex optimization problem and an approximate heuristic-based solution technique (based on Perron-Frobenius eigenvalue theory) is given. On the other hand, in our work, we present a new and simple unconstrained optimization based power control algorithm.

As we discussed earlier, in wireless networks, the QoS requirement of a particular Tx/Rx pair can be specified by certain minimum acceptable SINR. It can be assumed that the QoS requested is provided when the SINR exceeds a given threshold SINR ${ }^{\text {th }}$. Therefore, the outage probability of $i^{\text {th }}$ transmitter/receiver $\left(\mathrm{Tx} / \mathrm{Rx}_{i}\right)$ pair is written in alternative form as,

$$
O_{i}=\operatorname{Prob}\left(\operatorname{SINR}_{i} \leq \operatorname{SINR}^{t h}\right)
$$

Employing (2.11), outage probability in terms of all the transmitter powers and the 
channel gain parameters can be expressed as,

$$
O_{i}=\operatorname{Prob}\left(\gamma_{i i} G_{i i} P_{i} \leq \operatorname{SINR}^{t h}\left(\sum_{k \neq i} \gamma_{i k} G_{i k} P_{k}+\sigma^{2}\right)\right)
$$

It can be noted that the statistical variation of both received signal power and received interference power are taken into account in the expression for $O_{i}$. We can interpret the outage probability $O_{i}$ as the fraction of time the $i^{\text {th }}$ user experiences an outage due to channel variations.

Now, suppose $z_{i}, i=1,2, \cdots, N$ be the $N$ independent exponentially distributed random variables with mean $\mathbb{E}\left(z_{i}\right)=1 / \lambda_{i}$. Therefore, we can write the following probability identity,

$$
\operatorname{Prob}\left(z_{1}>\sum_{i=2}^{N} z_{i}+c\right)=e^{-\lambda_{1} c} \prod_{i=2}^{N}\left(\frac{1}{1+\frac{\lambda_{1}}{\lambda_{i}}}\right),
$$

where $c$ is a constant. A complete derivation of this relation is given in Appendix A.1.

Applying the above probability identity, the outage probability of $i^{\text {th }}$ transmitter/receiver can be written as,

$$
O_{i}=1-e^{-\frac{\sigma^{2} \operatorname{SINR} t h}{G_{i i} P_{i}}} \prod_{k \neq i} \frac{1}{\left(1+\frac{\operatorname{SINR}^{t h} G_{i k} P_{k}}{G_{i i} P_{i}}\right)} .
$$

In this section, our objective is to minimize outage probability subject to transmission power constraints. Putting $s_{i}=1 /\left(1-O_{i}\right)$, the above equation can be given in the following form,

$$
s_{i}=e^{\frac{\sigma^{2} \operatorname{SINR}^{t h}}{G_{i i} P_{i}}} \prod_{k \neq i}\left(1+\frac{\operatorname{SINR}^{t h} G_{i k} P_{k}}{G_{i i} P_{i}}\right) .
$$

Note that the outage probability in (3.5) can be minimized by minimizing $s_{i}$ in (3.6). Since logarithmic function is monotonically increasing, therefore, taking log on both side of (3.6), the objective function can be written as,

$$
t_{i}=\frac{\sigma^{2} \operatorname{SINR}^{t h}}{G_{i i} P_{i}}+\sum_{k \neq i} \log \left(1+\frac{\operatorname{SINR}^{t h} G_{i k} P_{k}}{G_{i i} P_{i}}\right) .
$$

where, $t_{i}=\log \left(s_{i}\right)$. In this work, we consider the optimization problem from system point of view. 


\subsection{Outage Based Optimal Power Control}

In this section, we formulate the optimal power control problem for maximizing network's interest by minimizing the outage probability of the system. We give an unconstrained optimization solution technique of the problem. This schemes optimize collective metrics for all users. Maximization of objectives like the sum of network information capacity [71], [72] or the sum of throughputs [73] falls into the networkcentric category. In a realistic scenario, the network has access to more global information than the mobile users. Therefore, it is more suitable for the network to play the role of decision maker [74].

In our case, for network-centric scheme, we try to minimize the system outage probability. The system outage probability is defined as the worst outage probability over all transmitter/receiver pairs. Mathematically, system outage probability is the maximum of the outage probabilities of the all transmitter/receiver pairs and can be given by,

$$
O=\max _{i} O_{i}
$$

The outage probability $O$ serves as a simple figure of merit for the system and power allocation.

In network-centric schemes, therefore, the system outage probability over all transmitter/receiver pairs is minimized with constraint on the transmission powers. The problem can be formulated as,

$$
\begin{array}{ll}
\operatorname{minimize} & O=\max _{i} O_{i} \\
\text { subject to } & O_{i}=1-e^{-\frac{\sigma^{2} \mathrm{SINR}^{t h}}{G_{i i} P_{i}}} \prod_{k \neq i} \frac{1}{\left(1+\frac{\operatorname{SINR}^{t h} G_{i k} P_{k}}{G_{i i} P_{i}}\right)} \\
& P_{i} \geq 0, i=1,2, \cdots, N
\end{array}
$$


With $t_{i}=\log \left(\frac{1}{1-O_{i}}\right)$, the problem becomes

$$
\begin{aligned}
\underset{i}{\operatorname{minimize} t=} & \max _{i} t_{i}, i=1,2, \cdots, N \\
= & \max _{i} \frac{\sigma^{2} \operatorname{SINR}^{t h}}{G_{i i} P_{i}}+\sum_{k \neq i} \log \left(1+\frac{\operatorname{SINR}^{t h} G_{i k} P_{k}}{G_{i i} P_{i}}\right) \\
\text { subject to } \quad & P_{i} \geq 0, i=1,2, \cdots, N
\end{aligned}
$$

It can be noted that with the transformation, the product term in (3.9) is converted into sum term in (3.10). Therefore, finding the expression for gradients become now easier, but the objective remaining the same.

Above constrained optimization problem can be converted into an unconstrained problem if we let $P_{i}=x_{i}^{2}$, where $x_{i}$ is any real number; and the optimization problem (3.10) can be written as,

$$
\begin{aligned}
\operatorname{minimize} t & =\max _{i} t_{i}, i=1,2, \cdots, N \\
& =\max _{i} \frac{\sigma^{2} \mathrm{SINR}^{t h}}{G_{i i} x_{i}^{2}}+\sum_{k \neq i} \log \left(1+\frac{\mathrm{SINR}^{t h} G_{i k} x_{k}^{2}}{G_{i i} x_{i}^{2}}\right)
\end{aligned}
$$

The problems becomes, therefore, a minimax problem.

The infinity-norm of an $N$-vector $\vec{t}=\left[t_{1}, t_{2}, \cdots, t_{N}\right]^{T}$ is defined as

$$
\|\vec{t}\|_{\infty}=\max _{i}\left(\left|t_{i}\right|, \text { for } 1 \leq i \leq N\right)
$$

Therefore, the optimization problem can be think of minimization of infinity-norm of vector $\vec{t}$.

If $p$ is even and the vector components are real numbers, then $p$-norm of vector $\vec{t}$ is defined as

$$
\|\vec{t}\|_{p}=\left(\sum_{i=1}^{N} t_{i}^{p}\right)^{\frac{1}{p}}
$$

The $p$-norm and infinity-norm are related by $\lim _{p \mapsto \infty}\|\vec{t}\|_{p}=\|\vec{t}\|_{\infty}$. Therefore, the optimization problem (3.11) can be expressed in terms of $p$-norm as follows:

$$
\operatorname{minimize} t=\lim _{p \rightarrow \infty}\left(\sum_{i=1}^{N} t_{i}^{p}\right)^{\frac{1}{p}}
$$


For even $p$, the $p$-norm of a vector is a differentiable function of its components, but the infinite norm is not. So, when the infinity norm is used in a problem, one can replace it by a $p$-norm. The difference between the approximate and exact solutions becomes negligible if the power $p$ is sufficiently large. The algorithm for.solving problem (3.14) is given in Table 3.1 which employs rank-two Davidon-Fletcher-Powell (DFP) method or Broyden-Fletcher-Goldfarb-Shanno (BFGS) method. The foundation of DFP and BFGS methods are classical Newton method and are referred to as quasi-Newton method (sometimes also called variable metric method). Unlike Newton method, quasi-Newton methods do not require explicit expression for the second order derivatives. The basic principle in quasi-Newton methods is that the direction of search is based on an $N \times N$ direction matrix $S$ which serves the same purpose as the inverse of Hessian in the Newton method. This matrix is generated from available data and is contrived to be an approximation of $H^{-1}$.Therefore, evaluation of Hessian Matrix and its inversion, which is computationally very expensive, are not required. Furthermore, as the number of iterations is increased, $S$ becomes progressively a more accurate representation of $H^{-1}$. Another advantage of DFP and BFGS methods are they do not need to check the positive definiteness in each iteration. Because, if $S_{k}$ is positive definite, then the matrix $S_{k+1}$ generated by these methods is also positive definite. However, they are almost as efficient as Newton method and quite tolerant to line search imprecision. The algorithm for finding optimal variable $\mathbf{x}=\left[x_{1}, x_{2}, \cdots, x_{N}\right]$ is described in Table 3.1. Complete DFP and.BFGS algorithms with expression for direction vector is given in Appendix A.2 and in Appendix A.3 respectively.

The gradient vector is defined as,

$$
\vec{g}_{i}(\vec{x})=\left[\frac{\delta t_{i}}{\delta x_{1}} \frac{\delta t_{i}}{\delta x_{2}} \cdots \frac{\delta t_{i}}{\delta x_{N}}\right]^{T}=\nabla t_{i}
$$

Therefore, the gradient vector of (3.14) is given by,

$$
\vec{g}(\vec{x})=\nabla t=\left[\frac{\delta t}{\delta x_{1}} \frac{\delta t}{\delta x_{2}} \cdots \frac{\delta t}{\delta x_{N}}\right]^{T}
$$




\section{Algorithm 1}

Step 1: Initialize optimization variable $\vec{x}^{(0)}$ and input tolerance $\epsilon$. Set iteration index $k=0$ and power $p=2$.

Step 2: Set direction matrix $\mathbf{S}^{(0)}=\mathbf{I}_{N}$, where $\mathbf{I}_{N}$ is a $N \times$ $N$ identity matrix. Compute gradient vector $\vec{g}^{(0)}$ at initial point.

Step 3: Compute direction vector using $\vec{d}^{(k)}=-\mathbf{S}^{(k)} \vec{g}^{(k)}$. Find $\alpha^{(k)}$ using a line search method. Set optimal direction vector $\vec{\delta}^{(k)}=\alpha^{(k)} \vec{d}^{(k)}$ and find updated value of variable $\vec{x}^{(k+1)}=\vec{x}^{(k)}+\vec{\delta}^{(k)}$.

Step 4: If $\left\|\vec{x}^{(k+1)}-\vec{x}^{(k)}\right\|<\epsilon$, then output $\vec{x}^{*}=\vec{x}^{(k+1)}, t^{*}=$ $\left(\sum_{i=1}^{N} t_{i}^{p}\right)^{\frac{1}{p}}$ and $O^{*}=1-e^{-t^{*}}$, and $P_{i}^{*}=\left(x_{i}^{*}\right)^{2}$, and stop.

Step 5: Compute gradient vector at updated point $\vec{g}^{(k+1)}$ and set $\vec{\beta}^{(k)}=\vec{g}^{(k+1)}-\vec{g}^{(k)}$. Compute direction matrix $\vec{S}^{(k+1)}$ using either DFP or BFGS algorithm described in Appendix A.2 or Appendix A.3 respectively with objective function given by (3.11) and gradient vector given by (3.17).

Step 6: Increase $k$ by 1 , double value of $p$ and repeat from Step 3

Table 3.1: Algorithm for solving outage-based power control to minimize system outage.

Combining (3.14)-(3.16) the expression for gradient vector (3.16) becomes, 


$$
\vec{g}(\vec{x})=\left(\sum_{i=1}^{N} t_{i}^{p}\right)^{\frac{1-p}{p}}\left[\begin{array}{cccc}
\frac{\delta t_{1}}{\delta x_{1}} & \frac{\delta t_{2}}{\delta x_{1}} & \cdots & \frac{\delta t_{N}}{\delta x_{1}} \\
\frac{\delta t_{1}}{\delta x_{2}} & \frac{\delta t_{2}}{\delta x_{2}} & \cdots & \frac{\delta t_{N}}{\delta x_{2}} \\
\vdots & \vdots & \ddots & \vdots \\
\frac{\delta t_{1}}{\delta x_{N}} & \frac{\delta t_{2}}{\delta x_{N}} & \cdots & \frac{\delta t_{N}}{\delta x_{N}}
\end{array}\right]\left[\begin{array}{c}
t_{1}^{p-1} \\
t_{2}^{p-1} \\
\vdots \\
t_{N}^{p-1}
\end{array}\right]
$$

where, for any $i$ and $k=1,2, \cdots, N$,

$$
\begin{aligned}
& \frac{\delta t_{i}}{\delta x_{i}}=-\frac{2 \sigma^{2} \operatorname{SINR}^{t h}}{G_{i i} x_{i}^{3}}-2 \operatorname{SINR}^{t h} \sum_{k \neq i} \frac{G_{i k} x_{k}^{2}}{x_{i}\left(G_{i i} x_{i}^{2}+\operatorname{SINR}^{t h} G_{i k} x_{k}^{2}\right)} \\
& \text { and } \quad \frac{\delta t_{i}}{\delta x_{k \neq i}}=2 \operatorname{SINR}^{t h} \frac{G_{i k} x_{k}}{\left(G_{i i} x_{i}^{2}+\operatorname{SINR}^{t h} G_{i k} x_{k}^{2}\right)}
\end{aligned}
$$

where, $\frac{\delta t_{i}}{\delta x_{k}}$ for $i, k=1,2, \cdots, N$ can be found from (3.18) and (3.19).

\subsection{Simulation Results}

We simulate some results for outage-based power control. In this section, we present those results and discussion.. Unless specified otherwise, the numerical data used for the simulation are given in Table 3.2. The optimal transmitted power for all the $\mathrm{Tx} / \mathrm{Rx}$ pairs using data given in Table 3.2 and $\operatorname{SINR}^{\text {th }}=7$ is shown in Table 3.4.

\begin{tabular}{|l|l|}
\hline Noise power, $\sigma^{2}$ & 0 \\
\hline $\begin{array}{l}\text { Cross gains are uniformly dis- } \\
\text { tributed with mean, } g_{\text {mean }}\end{array}$ & $5 \times 10^{-4}$ \\
\hline Self gain, $G_{i i}$ & 1 \\
\hline Number of users, $N$ & 50 \\
\hline SINR $^{\text {th }}$ & $3-11 \mathrm{~dB}$ \\
\hline
\end{tabular}

Table 3.2: Data used for simulation in outage-based power control

The variation of system outage probability with $\operatorname{SINR}^{\text {th }}$ for different mean cross gains is shown in Fig. 3.1. It is seen from the figure that the outage probability increases as SINR ${ }^{t h}$ increases. This fact is expected because as the value of the threshold 


\begin{tabular}{|l|l|l|l|l|l|}
\hline $\begin{array}{l}\text { Tx/Rx } \\
\text { pair }\end{array}$ & $\begin{array}{l}\text { Optimal } \\
\text { power }\end{array}$ & $\begin{array}{l}\text { Tx/Rx } \\
\text { pair }\end{array}$ & $\begin{array}{l}\text { Optimal } \\
\text { power }\end{array}$ & $\begin{array}{l}\text { Tx/Rx } \\
\text { pair }\end{array}$ & $\begin{array}{l}\text { Optimal } \\
\text { power }\end{array}$ \\
\hline 1 & 1.0175 & 18 & 0.9992 & 35 & 0.9844 \\
\hline 2 & 1.0473 & 19 & 1.0412 & 36 & 1.0243 \\
\hline 3 & 1.0818 & 20 & 0.8760 & 37 & 0.9871 \\
\hline 4 & 0.9213 & 21 & 0.9788 & 38 & 1.0098 \\
\hline 5 & 0.9773 & 22 & 0.9625 & 39 & 1.0992 \\
\hline 6 & 1.0053 & 23 & 0.9457 & 40 & 0.0924 \\
\hline 7 & 0.8286 & 24 & 1.0458 & 41 & 0.9992 \\
\hline 8 & 0.8693 & 25 & 0.9412 & 42 & 1.0633 \\
\hline 9 & 1.1148 & 26 & 0.9816 & 43 & 1.0774 \\
\hline 10 & 1.0403 & 27 & 0.9908 & 44 & 0.9976 \\
\hline 11 & 1.0256 & 28 & 1.1493 & 45 & 0.9849 \\
\hline 12 & 0.9925 & 29 & 1.0301 & 46 & 0.8746 \\
\hline 13 & 0.9240 & 30 & 1.0096 & 47 & 0.9970 \\
\hline 14 & 0.9202 & 31 & 0.9889 & 48 & 0.9405 \\
\hline 15 & 1.1794 & 32 & 1.0224 & 49 & 1.1170 \\
\hline 16 & 0.9682 & 33 & 0.9861 & 50 & 0.9869 \\
\hline 17 & 0.9286 & 34 & 1.0023 & - & - \\
\hline & & & & & \\
\hline
\end{tabular}

Table 3.3: Optimal transmitted power vector of system outage probability minimization problem for particular data 
increases, the probability of instantaneous SINR being less than or equal to that threshold increases. According to the definition of outage probability, this in turn increases the outage probability. Increased value of mean cross gain means increased interference power. Therefore, the instantaneous SINR decreases as mean cross-gain increases. This effect is shown in this figure also. It can be noted that as the mean cross gain increases, the outage increases. It has also been observed that as cross gain increases, the slope of the curve increases.

In Fig. 3.2, we show the effect of number of users on the system outage probability. It is seen that as the number of user increases, the system outage probability also increases. Since the interference power increases with number of users, hence this results is justified.

The dependence of total power on the number of transmitter/receiver pairs for network-centric case is shown in Fig. 3.3. The total power is found to increase with the number of $\mathrm{Tx} / \mathrm{Rx}$ pairs. This effect can be explained by understanding the fact that as number of $\mathrm{Tx} / \mathrm{Rx}$ increases, the component of interferer and hence the total interference power increases. Therefore, to minimize the outage, the transmitter has to use larger transmission power. It is also found that the total power does not depend on the SINR ${ }^{\text {th }}$ and mean-cross gain. Because whatever the SINR ${ }^{t h}$ may be, it affect only outage for given power. 


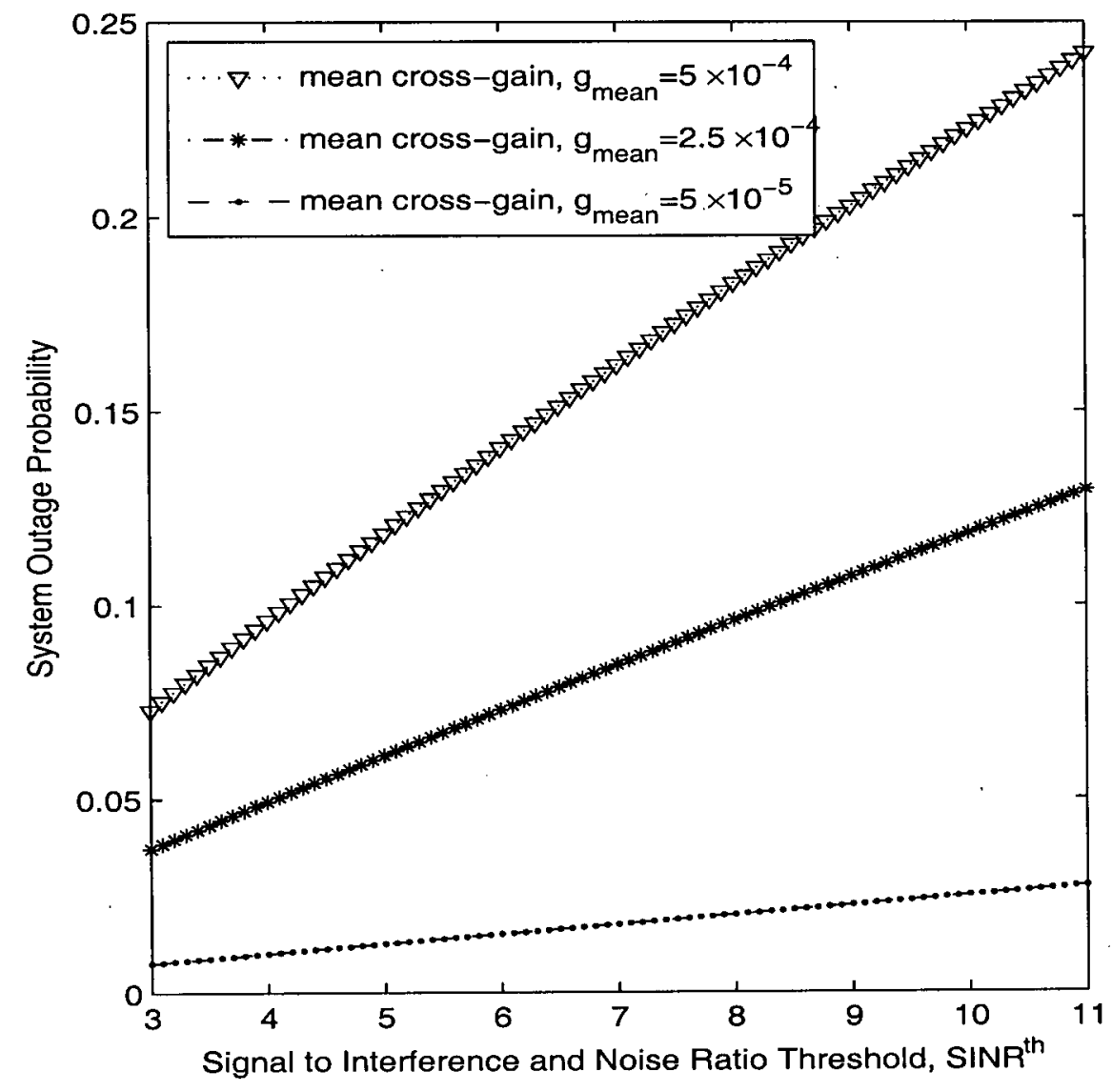

Figure 3.1: Variation of system outage probability with SINR $^{\text {th }}$ for different mean cross-gain. 


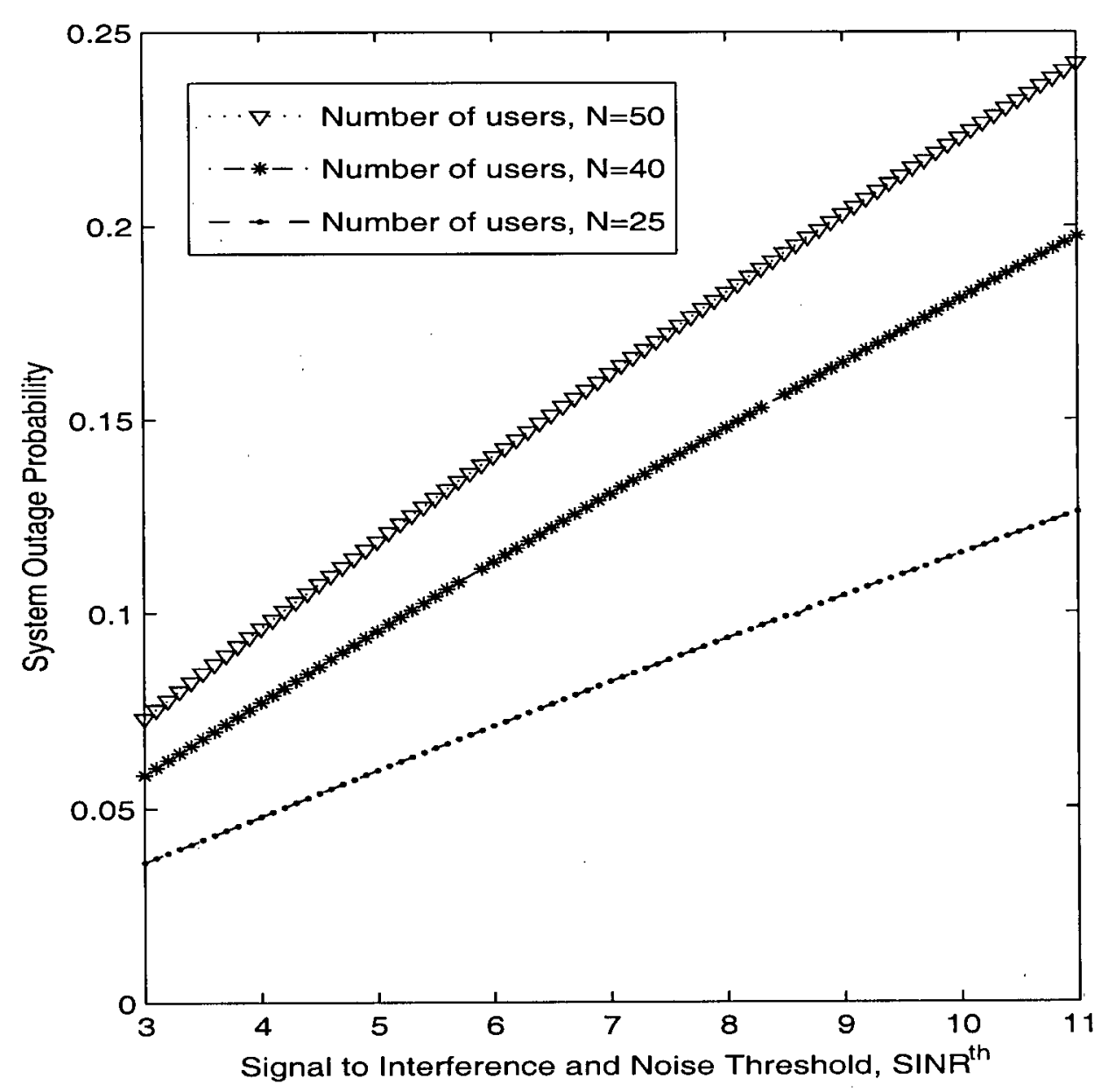

Figure 3.2: Influence of number of transmitter/receiver pairs on system outage probability vs. SINR ${ }^{\text {th }}$ curve. 




Figure 3.3: Dependence of total power on the number of transmitter/receiver pairs 


\section{Chapter 4}

\section{Utility-based Power Control}

\subsection{Introduction}

Over the past decade, the tremendous growth in the mobile internet user population has been accompanied by an equally exciting evolution in wireless data networks. The next generation of wireless systems promise to offer high speed delivery of multimedia information integrated with voice and data. In the previous chapter, we have discussed outage-based optimal power control algorithms for interference-limited wireless networks. The QoS requirements for different user is given in terms of outage probability. The QoS objective for a voice terminal is to achieve a minimum acceptable SINR and therefore outage based optimal power control is more appropriate for wireless voice systems. However, this approach is not suitable for the efficient operation of wireless data systems. This is because the QoS objective for data signals differs from the QoS objective for telephones. In telephone systems, low delay is essential, and transmission errors are tolerable upto a point. By contrast, data signals can accept some delay but have very low tolerance to errors. The QoS requirements of data communications are given more appropriately with utility function. In this chapter, we propose two optimal power control algorithms for interference-limited wireless networks based on the utility function [1]. In Section 4.2, we explain why utility based power control is more ap- 
propriate for data networks. The user-centric and network-centric utility based power control algorithms and their solution techniques are given in Section 4.3.1 and Section 4.3.2 respectively. In Section 4.4, we show some simulation results.

\subsection{Utility}

Second-generation (2G) and third-generation (3G) wireless systems have been designed primarily for voice, a connection-oriented and delay sensitive service requiring a specified bit rate. The tremendous growth in the mobile Internet user population has been accompanied by an equally exciting evolution in wireless data networks with different QoS requirements. In contrast to voice, data services are often connectionless and delay insensitive, and have no specific bit-rate requirements. These differences suggest that ubiquitous (anytime/anywhere) coverage may not be a strict requirement for wireless data networks (and in fact needlessly complicate the design) [75]. Therefore, to more efficiently accommodate different services with different characteristics, a new frame work has been proposed in [76]-[78] using the concepts from microeconomics.

In economics, utility is defined as the level of satisfaction that a person (user) derives from consuming a good or undertaking an activity. Formally, a utility function is defined as follows [79], [80].

Definition 1: A function that assigns a numerical value to the elements of the action set $A\left(u: \rightarrow \mathbf{R}^{\mathbf{1}}\right)$ is a utility function, if, for all $x, y \in A, x$ is at least as preferred compared to $y$ if and only if $u(x) \geq u(y)$.

The utility function that describes a particular set of preference rules is not unique. Any function that puts the elements of $A$ in the desired order is a candidate for utility function.

In the wireless communications literature, QoS is closely related to utility. Two QoS objectives are low delay and low probability of error. In telephone systems low delay is essential, and transmission errors are tolerable up to a point. Therefore, for 
voice communications the acceptable quality of connection is often specified by some maximum tolerable BER (usually $10^{-3}$ ) or minimum SINR requirement. Above this required SINR, the voice user has an acceptable connection (utility "one") and below this threshold the voice user does not have a satisfactory voice quality (utility "zero"). The reason behind this zero-one property of voice utility is anything better than the required SINR is virtually the same for human ear[81]. In cellular telephone systems, the target SINR threshold is system-dependent. For code-division multiple access it is on the order of $6 \mathrm{~dB}[7]$.

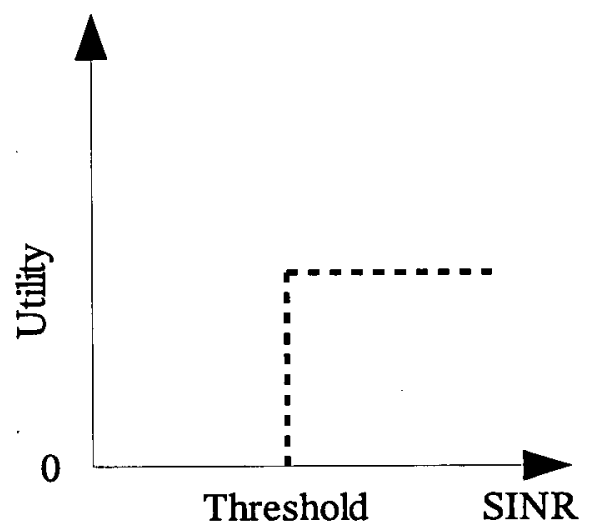

(a)
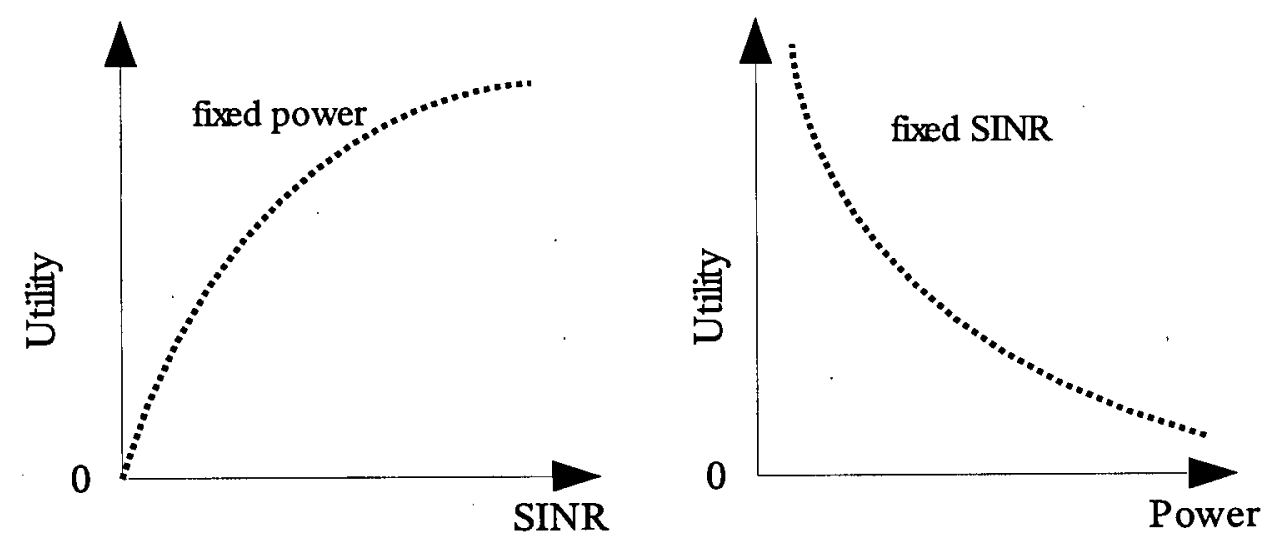

(b)

Figure 4.1: Conceptual utility for (a) voice and (b) data

By contrast, data signals can accept some delay but have very low tolerance to 
errors (BER on the order of $10^{-6}$ or lower). Further, data users generally communicate information in form of packets. When a system contained FEC coding, transmission error appears at the output of the FEC decoder. Due to their intolerance of errors, a packet containing errors will have to be retransmitted, since error correction coding can correct only a few errors. A higher SINR will lead to lesser number of retransmission, and hence reduce the traffic delay experienced by the data user. Thus for a data user, a higher SINR generally implies a better throughput. This implies that the utility function for a data user is a continuous function of the SINR obtained by the user [76]. A conceptual plot of the utilities of a voice user and a data user is given in Fig. 4.1.

In addition to the speed of data transfer, a factor in the utility of data systems, power consumption is an important factor in mobile computing. Thus, we see that utility depends on both SINR and transmit power. Of course, these quantities are interdependent. If the transmit power were fixed (fixed battery drain), the terminal would experience lower error rates as SINR increases which leads to increased satisfaction of the use of the system resources. For sufficiently large SINR values, the error rate approaches to zero which results in an asymptotic increase in utility in the high SINR region. If the SINR were to be fixed (fixed error rate), increasing the transmit power expedites the battery drain, which effectively reduces the satisfaction of the mobile terminal [6]. Hence, services with heterogeneous QoS requirements can be modeled with different utility functions.

\subsection{Utility-based Optimal Power Control}

For data communications, information is usually transmitted in the form of packets or frames. The measure of satisfaction derived from using a mobile data system clearly is related to the amount of information that a user can transfer in the lifetime of its battery. Thus, the utility function for data system is defined as the number of information bits received successfully per Joule of energy expended [51]. Let us assume that data bits 
are packed into frames of $F$ bits containing $L$ information bits and $F-L$ parity bits for error detection. The transmission rate for all transmitter/receiver pair is fixed to $R_{s}$ symbols/second. Therefore, the bit transmission rate of the transmitter is $R=R_{s} k$, where $k$ is the number of bits/symbols. The utility function of $i^{\text {th }}$ transmitter/receiver can be given by,

$$
u_{i}=\frac{L R f_{e f f}\left(\mathrm{SINR}_{i}\right)}{F P_{i}}
$$

where $f_{\text {eff }}\left(\mathrm{SINR}_{i}\right)$ is efficiency function that closely approximates the frame success

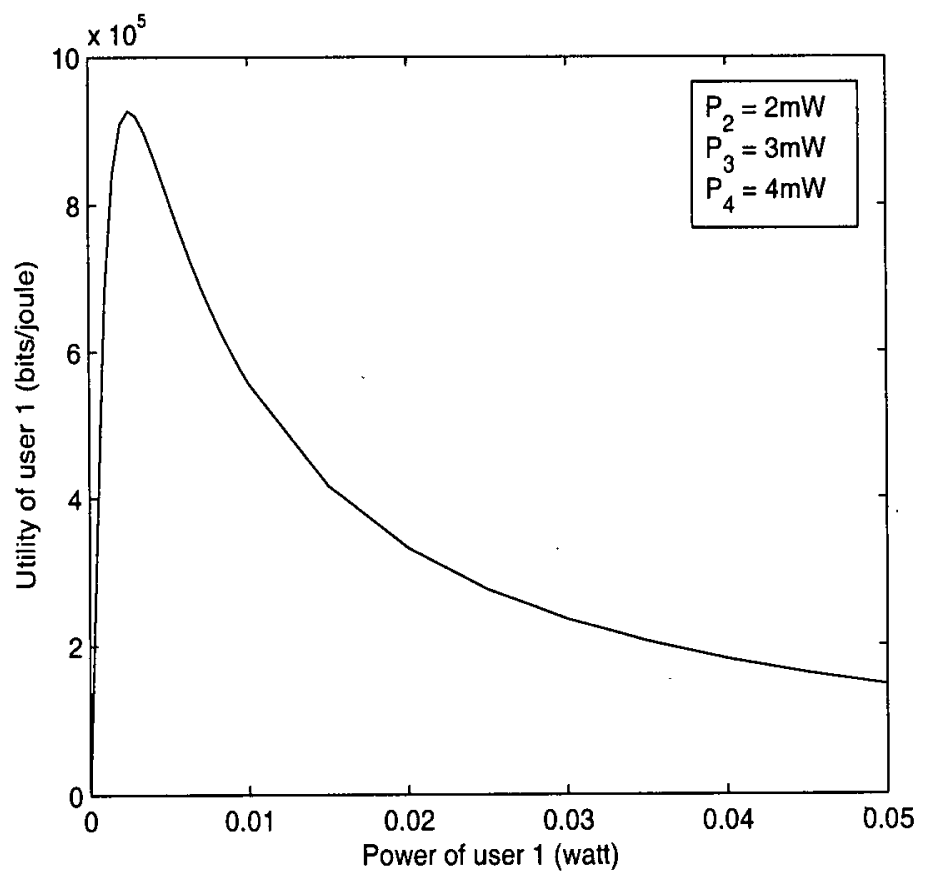

Figure 4.2: Typical variation of individual utility with its transmitted power

rate (FSR) with the properties $f_{\text {eff }}(\infty)=1$ and $\frac{f_{\text {eff }}\left(\mathrm{SINR}_{i}\right)}{P_{i}}=0$ for $P_{i}=0$. The efficiency function can be expressed as $f_{\text {eff }}\left(\operatorname{SINR}_{i}\right)=\left(1-O_{i}\right)$ [82], where $O_{i}$ is the frame outage probability and given by (3.5). Therefore, we can express the utility function for the $i$ th transmitter/receiver pair as,

$$
u_{i}=\frac{L R}{F P_{i}} e^{-\frac{\sigma^{2} \mathrm{SINR}^{t h}}{G_{i i} P_{i}}} \prod_{k \neq i} \frac{1}{\left(1+\frac{\operatorname{SINR}^{t h} G_{i k} P_{k}}{G_{i i} P_{i}}\right)}
$$


Fig. 4.2 shows the nature of the utility function as a function of its own transmission power when others' transmitted powers are kept fixed. In this case, we consider a system with 4 users and plot the utility of user $i$ with power $P_{i}$. The powers for the three remaining users in the system are kept fixed. It can be noted that $u_{i}$ goes to zero as $p_{i} \rightarrow 0$ or $p_{i} \rightarrow \infty$. That means there is a unique maximum and the function is strictly convex.

Letting $t_{i}=\log \left(1 / u_{i}\right)$, equation (4.2) can be rewritten as,

$$
t_{i}=\log \left(\frac{F P_{i}}{L R}\right)+\frac{\sigma^{2} \mathrm{SINR}^{t h}}{G_{i i} P_{i}}+\sum_{k \neq i} \log \left(1+\frac{\mathrm{SINR}^{t h} G_{i k} P_{k}}{G_{i i} P_{i}}\right)
$$

It is clear that maximizing utility function $u_{i}$ is equivalent to minimizing function $t_{i}$. The SINR ${ }^{\text {th }}$ depends on the modulation used and specified minimum FSR.

The FSR of $\mathrm{Tx} / \mathrm{Rx}_{i}$ is the probability of all bits in a frame being received correctly and can be expressed as,

$$
\mathrm{FSR}_{i}=\left(1-P_{e_{i}}\right)^{F}
$$

where $P_{e_{i}}$ is the bit error rate of modulation used for transmission.

- BER for BPSK Transmission: For BPSK transmission, the BER can be given as [83],

$$
P_{e_{i}}=\frac{1}{2} \operatorname{erfc}\left(\sqrt{\operatorname{SINR}_{i}}\right)
$$

Combining (4.4) and (4.5), the $\mathrm{SINR}_{i}$ can be expressed as,

$$
\operatorname{SINR}_{i}=\left(\operatorname{erfc}^{-1}\left(2 P_{e_{i}}\right)\right)^{2}=\left(\operatorname{erfc}^{-1}\left(2\left(1-\left(\mathrm{FSR}_{i}\right)^{1 / F}\right)\right)\right)^{2}
$$

where $\operatorname{erfc}^{-1}($.$) is the inverse complementary error function.$

- BER Approximation for M-QAM Transmission: The expression for the BER of square M-quadrature amplitude modulation (M-QAM) with Gray bit mapping in additive white Gaussian noise (AWGN) as a function of received $\operatorname{SINR}_{i}$ and constellation $M=2^{k}$ size is approximately [83],

$$
P_{e_{i}} \approx \frac{2}{k}\left(1-\frac{1}{\sqrt{2^{k}}}\right) \times \operatorname{erfc}\left(\sqrt{1.5 \frac{\operatorname{SINR}_{i}}{2^{k}-1}}\right)
$$


The approximation of BER for M-QAM transmission tight to within $1 \mathrm{~dB}$ for $k \geq 2$ and BER $\leq 10^{-3}$ is given by [84],

$$
P_{e_{i}} \approx \frac{1}{5} \exp \left(-\frac{1.6 \mathrm{SINR}_{i}}{M-1}\right)
$$

Hence, the SINR for $\mathrm{Tx} / \mathrm{Rx}_{i}$ can be expressed as,

$$
\mathrm{SINR}_{i} \approx \frac{1-M}{1.6} \log \left(5 P_{e_{i}}\right)=\frac{1-M}{1.6} \log \left(5\left(1-\left(\mathrm{FSR}_{i}\right)^{1 / F}\right)\right) .
$$

- BER Approximation for M-PSK Transmission: The BER expression for M-phase shift keying (M-PSK) in AWGN with Gray bit mapping and $M=2^{k}$ is commonly approximated as [83],

$$
P_{e_{i}} \approx \frac{1}{k} \operatorname{erfc}\left(\sqrt{\operatorname{SINR}_{i}} \sin \left(\frac{\pi}{2^{k}}\right)\right)
$$

The approximate BER that are valid for $k \geq 2$ within $1.5 \mathrm{~dB}$ of error for $\mathrm{BER} \leq$ $10^{-3}$ is given by,

$$
P_{e_{i}} \approx \frac{1}{5} \exp \left[\frac{7 \operatorname{SINR}_{i}}{2^{1.9 k}+1}\right]
$$

Hence, the SINR for $\mathrm{Tx} / \mathrm{Rx}_{i}$ can be expressed as,

$$
\operatorname{SINR}_{i} \approx \frac{1+2^{1.9 k}}{7} \log \left(5 P_{e_{i}}\right)=\frac{1+2^{1.9 k}}{7} \log \left(5\left(1-\left(\mathrm{FSR}_{i}\right)^{1 / F}\right)\right)
$$

Therefore, for specified FSR, the SINR ${ }^{\text {th }}$ can be obtained from (4.6), (4.9), or (4.12).

In the sequel, we discuss two optimal power control algorithm from the view point of individual user's interest and overall network's interest with the goal of maximizing utility. We also give the unconstrained optimization-based solutions of these two problems.

\subsubsection{User-centric Case}

In this case, the main objective of each individual user is to maximize its QoS quantified through the utility function by unilaterally adjusting its transmit power. Therefore, 
for user-centric scheme, the objective is to maximize utility of individual $i^{\text {th }}$ transmitter/receiver pair with the constraints that all the transmitter power is non negative. The problem can be formulated as follows:

$$
\begin{array}{ll}
\operatorname{maximize} & u_{i} \\
\text { subject to } & u_{i}=\frac{L R}{F P_{i}} e^{-\frac{\sigma^{2} \operatorname{SINR} R^{t h}}{G_{i i} P_{i}}} \prod_{k \neq i} \frac{1}{\left(1+\frac{\operatorname{SINR}^{t h} G_{i k} P_{k}}{G_{i i} P_{i}}\right)} \\
& P_{i} \geq 0, i=1,2, \cdots, N
\end{array}
$$

Eliminating the nonnegativity bound by replacing $P_{i}=x_{i}^{2}$ and applying $t_{i}=\log \left(1 / u_{i}\right)$, we get the following unconstrained minimization problem,

$$
\operatorname{minimize} t_{i}=\log \left(\frac{F x_{i}^{2}}{L R}\right)+\frac{\sigma^{2} \operatorname{SINR}^{t h}}{G_{i i} x_{i}^{2}}+\sum_{k \neq i} \log \left(1+\frac{\operatorname{SINR}^{t h} G_{i k} x_{k}^{2}}{G_{i i} x_{i}^{2}}\right)
$$

The problem derived in final form (4.14) can be solved using either DFP and BFGS methods. We give the algorithms in Table 4.1. The expression for gradient vector is given by,

$$
\vec{g}_{i}(\vec{x})=\nabla t_{i}=\left[\frac{\delta t_{i}}{\delta x_{1}} \frac{\delta t_{i}}{\delta x_{2}} \cdots \frac{\delta t_{i}}{\delta x_{N}}\right]^{T}
$$

where, for any $i$ and $k=1,2, \cdots, N$,

$$
\begin{aligned}
& \frac{\delta t_{i}}{\delta x_{i}}=\frac{2}{x_{i}}-\frac{2 \sigma^{2} \operatorname{SIR}^{t h}}{G_{i i} x_{i}^{3}}-2 \operatorname{SINR}^{t h} \sum_{k \neq i} \frac{G_{i k} x_{k}^{2}}{x_{i}\left(G_{i i} x_{i}^{2}+\operatorname{SINR}^{t h} G_{i k} x_{k}^{2}\right)} \\
& \text { and } \quad \frac{\delta t_{i}}{\delta x_{k \neq i}}=2 \operatorname{SINR}^{t h} \frac{G_{i k} x_{k}}{\left(G_{i i} x_{i}^{2}+\operatorname{SINR}^{t h} G_{i k} x_{k}^{2}\right)}
\end{aligned}
$$

\subsubsection{Network-centric Case}

In this scheme, a central controller keeps the track of the entire network of interfering transmission and their utilities. Our goal in this problem is to maximize the minimum 


\section{Algorithm 2}

Step 1: $\quad$ Set iteration index $k=0$ and direction matrix $\mathbf{S}^{(0)}=$ $\mathbf{I}_{N}$, where $\mathbf{I}_{N}$ is a $N \times N$ identity matrix. Initialize optimization variable $\vec{x}^{(0)}$ and compute gradient vector $\vec{g}^{(0)}$ at initial point. Input tolerance $\epsilon$.

Step 2: Find $\alpha^{(k)}$ using a line search method and compute direction vector using $\vec{d}^{(k)}=-\mathbf{S}^{(k)} \vec{g}^{(k)}$. Set optimal direction vector $\vec{\delta}^{(k)}=\alpha^{(k)} \vec{d}^{(k)}$ and find updated value of variable $\vec{x}^{(k+1)}=\vec{x}^{(k)}+\vec{\delta}^{(k)}$.

Step 3: If $\left|\vec{\delta}^{(k)}\right|<\epsilon$, then output $\vec{x}^{*}=\vec{x}^{(k+1)}, t_{i}^{*}=t_{i}\left(\vec{x}^{(k+1)}\right)$, $u_{i}^{*}=e^{-t_{i}^{*}}$ and $P_{i}^{*}=\left(x_{i}^{*}\right)^{2}$, and stop.

Step 4: Compute gradient vector at updated point $\vec{g}^{(k+1)}$ and set $\vec{\beta}^{(k)}=\vec{g}^{(k+1)}-\vec{g}^{(k)}$. Compute direction matrix $\vec{S}^{(k+1)}$ using either DFP or BFGS algorithm described in Appendix A.2 or Appendix A.3 respectively with objective function given by (4.14) and gradient vector given by (4.15). Set $k=k+1$ and repeat from Step 2.

Table 4.1: Algorithm for solving utility-based power control in user-centric case utility over all transmitter/receiver pairs with transmission power constraint as,

$$
\begin{array}{ll}
\operatorname{maximize} & U=\min _{i} u_{i} \\
\text { subject to } & u_{i}=\frac{L R}{F P_{i}} e^{-\frac{\sigma^{2} \operatorname{SINR}_{i i} P_{i}}{G_{i}}} \prod_{k \neq i} \frac{1}{\left(1+\frac{\operatorname{SINR}^{t h} G_{i k} P_{k}}{G_{i i} P_{i}}\right)} \\
P_{i} \geq 0, i=1,2, \cdots, N
\end{array}
$$




\begin{tabular}{|c|c|}
\hline & Algorithm 3 \\
\hline Step 1: & $\begin{array}{l}\text { Set iteration index } k=0 \text { and power } p=2 \text {. Initialize } \\
\text { optimization variable } \vec{x}^{(0)} \text {. Input tolerance } \epsilon .\end{array}$ \\
\hline Step 2: & $\begin{array}{l}\text { Using either DFP or BFGS algorithm described in } \\
\text { Appendix A.2 or Appendix A.3 respectively, find } \\
\vec{x}^{(k+1)} \text { with objective function given by }(4.19) \text { and } \\
\text { gradient vector given by }(4.20) \text {. }\end{array}$ \\
\hline Step 3: & $\begin{array}{l}\text { If }\left\|\vec{x}^{(k+1)}-\vec{x}^{(k)}\right\|<\epsilon \text {, then output } \vec{x}^{*}=\vec{x}^{(k+1)}, t^{*}= \\
\left(\sum_{i=1}^{N} t_{i}^{p}\right)^{\frac{1}{p}} \text { and } U^{*}=e^{-t^{*}}, \text { and stop. }\end{array}$ \\
\hline Step 4: & Set $k=k+1, p=2 p$ and repeat from Step 2 \\
\hline
\end{tabular}

Table 4.2: Algorithm for solving utility-based power control in network-centric case After applying similar transformation techniques discussed in Section 4.3.1, i.e., $P_{i}=$ $x_{i}^{2}$ and $t_{i}=\log \left(1 / u_{i}\right)$, the new formulation that is more suitable for solving can be given by,

$$
\operatorname{minimize} t=\max _{i} \log \left(\frac{F x_{i}^{2}}{L R}\right)+\frac{\sigma^{2} \operatorname{SINR}^{t h}}{G_{i i} x_{i}^{2}}+\sum_{k \neq i} \log \left(1+\frac{\operatorname{SINR}^{t h} G_{i k} x_{k}^{2}}{G_{i i} x_{i}^{2}}\right)
$$

Since this problem becomes a minimax problem, we use the concept of $p$-norm and infinite-norm discussed in Section 3.3 to get the following equivalent problem,

$$
\operatorname{minimize} t=\lim _{p \rightarrow \infty}\left(\sum_{i=1}^{N} t_{i}^{p}\right)^{\frac{1}{p}}
$$

where, $t_{i}$ is given by (4.3). With objective function (4.19), we can solve the above problem using either DFP or BFGS algorithm. We give the algorithm in Table 4.2. The 
gradient vector for this algorithm can be given as,

$$
\vec{g}(\vec{x})=\left(\sum_{i=1}^{N} t_{i}^{p}\right)^{\frac{1-p}{p}}\left[\begin{array}{cccc}
\frac{\delta t_{1}}{\delta x_{1}} & \frac{\delta t_{2}}{\delta x_{1}} & \cdots & \frac{\delta t_{N}}{\delta x_{1}} \\
\frac{\delta t_{1}}{\delta x_{2}} & \frac{\delta t_{2}}{\delta x_{2}} & \cdots & \frac{\delta t_{N}}{\delta x_{2}} \\
\vdots & \vdots & \ddots & \vdots \\
\frac{\delta t_{1}}{\delta x_{N}} & \frac{\delta t_{2}}{\delta x_{N}} & \cdots & \frac{\delta t_{N}}{\delta x_{N}}
\end{array}\right]\left[\begin{array}{c}
t_{1}^{p-1} \\
t_{2}^{p-1} \\
\vdots \\
t_{N}^{p-1}
\end{array}\right]
$$

where, $\frac{\delta t_{i}}{\delta x_{k}}$ for $i, k=1,2, \cdots, N$ can be found from (4.16) and (4.17).

\subsection{Simulation Results}

In this section, we present some simulation results for both the user-centric and the network-centric utility-based power control. The experimental data are given in Table 4.3.The optimal power of all $\mathrm{Tx} / \mathrm{Rx}$ pair for network-centric scheme with data given in Table 4.3 and FSR $=0.999$ is shown in Table 4.4 .

\begin{tabular}{|l|l|}
\hline Modulation used & $\begin{array}{l}4,16,64-\mathrm{QAM}, \\
2,4,8,16,32,64-\mathrm{PSK}\end{array}$ \\
\hline Noise power $\sigma^{2}$ & $5 \times 10^{-5}$ \\
\hline Packet size, $F$ & 80 bits \\
\hline Information bits/packet, $L$ & 64 bits \\
\hline Mean cross gain, $g_{\text {mean }}$ & $5 \times 10^{-4}$ bits \\
\hline Frame success rate, FSR & $0.9900-0.9999$ bits \\
\hline Number of users, $N$ & 50 \\
\hline SINR ${ }^{\text {th }}$ & $3-11 \mathrm{~dB}$ \\
\hline Transmission rate, $R_{s}$ & $10^{4}$ symbols $/ \mathrm{sec}$ \\
\hline
\end{tabular}

Table 4.3: Data used for simulation in utility-based power control

In Fig. 4.3(a), we show the variation of individual $\mathrm{Tx} / \mathrm{Rx}$ utility for user-centric case with SINR $^{t h}$. It is seen that the utility decreases as SINR ${ }^{t h}$ increases. This is 


\begin{tabular}{|c|c|c|c|c|c|}
\hline $\begin{array}{l}\mathrm{Tx} / \mathrm{Rx} \\
\text { pair }\end{array}$ & $\begin{array}{l}\text { Optimal } \\
\text { power }\end{array}$ & $\begin{array}{l}\mathrm{Tx} / \mathrm{Rx} \\
\text { pair }\end{array}$ & $\begin{array}{l}\text { Optimal } \\
\text { power }\end{array}$ & $\begin{array}{l}\mathrm{Tx} / \mathrm{Rx} \\
\text { pair }\end{array}$ & $\begin{array}{l}\text { Optimal } \\
\text { power }\end{array}$ \\
\hline 1 & 0.0484 & 18 & 0.0491 & 35 & 0.0472 \\
\hline 2 & 0.0471 & 19 & 0.0495 & 36 & 0.0457 \\
\hline 3 & 0.0537 & 20 & 0.0487 & 37 & 0.0531 \\
\hline 4 & 0.0560 & 21 & 0.0564 & 38 & 0.0490 \\
\hline 5 & 0.0471 & 22 & 0.0512 & 39 & 0.0438 \\
\hline 6 & 0.0475 & 23 & 0.0552 & 40 & 0.0471 \\
\hline 7 & 0.0435 & 24 & 0.0687 & 41 & 0.0444 \\
\hline 8 & 0.0496 & 25 & 0.0440 & 42 & 0.0528 \\
\hline 9 & 0.0520 & 26 & 0.0541 & 43 & 0.0471 \\
\hline 10 & 0.0526 & 27 & 0.0499 & 44 & 0.0489 \\
\hline 11 & 0.0522 & 28 & 0.0462 & 45 & 0.0490 \\
\hline 12 & 0.0466 & 29 & 0.0524 & 46 & 0.0449 \\
\hline 13 & 0.0506 & 30 & 0.0494 & 47 & 0.0573 \\
\hline 14 & 0.0554 & 31 & 0.0561 & 48 & 0.0435 \\
\hline 15 & 0.0512 & 32 & 0.0567 & 49 & 0.0443 \\
\hline 16 & 0.0526 & 33 & 0.0525 & 50 & 0.0512 \\
\hline 17 & 0.0516 & 34 & 0.0482 & - & - \\
\hline
\end{tabular}

Table 4.4: Optimal transmitted power vector of system utility maximization problem for particular data 
because as SINR ${ }^{\text {th }}$ increases, outage increases, and therefore utility decreases. The FSR increases as the SINR ${ }^{\text {th }}$ increases. This is due to the fact that as $\operatorname{SINR}^{\text {th }}$ increases, the probability of bit error decreases, this in turn increases FSR. Since for the same $\mathrm{SINR}^{\text {th }}$, the bit error probability of higher order modulation is more, therefore, higher order modulation has lesser FSR.

We plot the influence of FSR on individual Tx/Rx pair utility in Fig. 4.4-4.5 and Fig. 4.6 for different MPSK and different MQAM modulation respectively. It is seen that the utility decreases as FSR increases. This fact can be explained with the result shown in Fig. 4.3. For increased FSR, we need to increase SINR ${ }^{\text {th }}$, this in turn decreases the utility. It can be also note that the utility of higher modulation is less than lower order modulation. Again, since for same FSR, we need larger SINR ${ }^{\text {th }}$ for higher order modulation, the noted effect is justified. Another point to be noted from the curves is the rate of decrease of utility is more as FSR increases.

The next three plots, i.e., Fig. 4.7-4.8 and Fig. 4.9, show the variations of total transmission power with FSR for different MPSK and different MQAM modulation respectively. We see that the power increases as FSR increases. Because as FSR for particular modulation increases, the SINR ${ }^{\text {th }}$ also increases, and hence the outage increases. Therefore, to minimize the outage probability, the individual transmitter power increases. Since higher order modulation necessitates larger $\operatorname{SINR}^{\text {th }}$, this increases outage and correspondingly transmission power.

The last five plots (Fig. 4.10-4.14) shows the result for the network-centric case. In Fig. 4.10, we show the variation of system utility with frame success rate for MPSK modulation. We have observed that the system utility decrease as the FSR increase. That means, FSR has the similar type of effect on system utility as in the case of individual TX/Rx pair utility.

In Fig. 4.11, we plot the effect of number of $\mathrm{Tx} / \mathrm{Rx}$ pairs on system utility: - It is seen that as the number of $\mathrm{Tx} / \mathrm{Rx}$ pairs increases, the system utility decreases. The reason behind this can be explained by understanding the fact that as the number of 
transmitter/receiver pairs increases, the component of cross gain increases which in turn increases the interference and reduce the utility.

The dependence of system utility on the noise power is shown in Fig. 4.12. It has been seen that as the noise power increases, the utility decreases; which is obvious.

We plot the variation of total power with FSR for network-centric case in Fig. 4.13. We observe the same type of result as for the user-centric case, i.e. total power increases as FSR increases. The fact behind this is same as individual utility.

Fig. 4.14, shows the influence of number of transmitter/receiver pairs on total power. The total power increases at a constant rate with respect to number of $T x / R x$ pairs. The is because as the number of $\mathrm{Tx} / \mathrm{Rx}$ pair increases, the component of interferer and hence total interference power increases. Therefore to maximize the system utility, transmission power has to increase. 




(a)

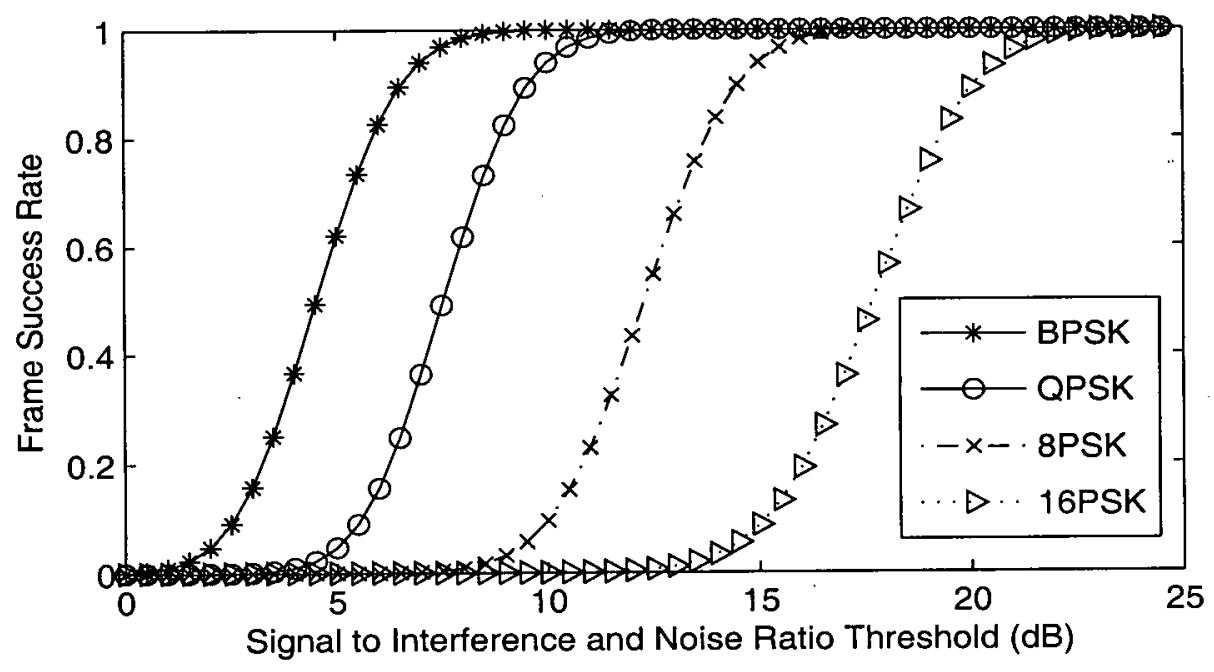

(b)

Figure 4.3: (a) Variation of utility (b) frame success rate with SINR threshold. 


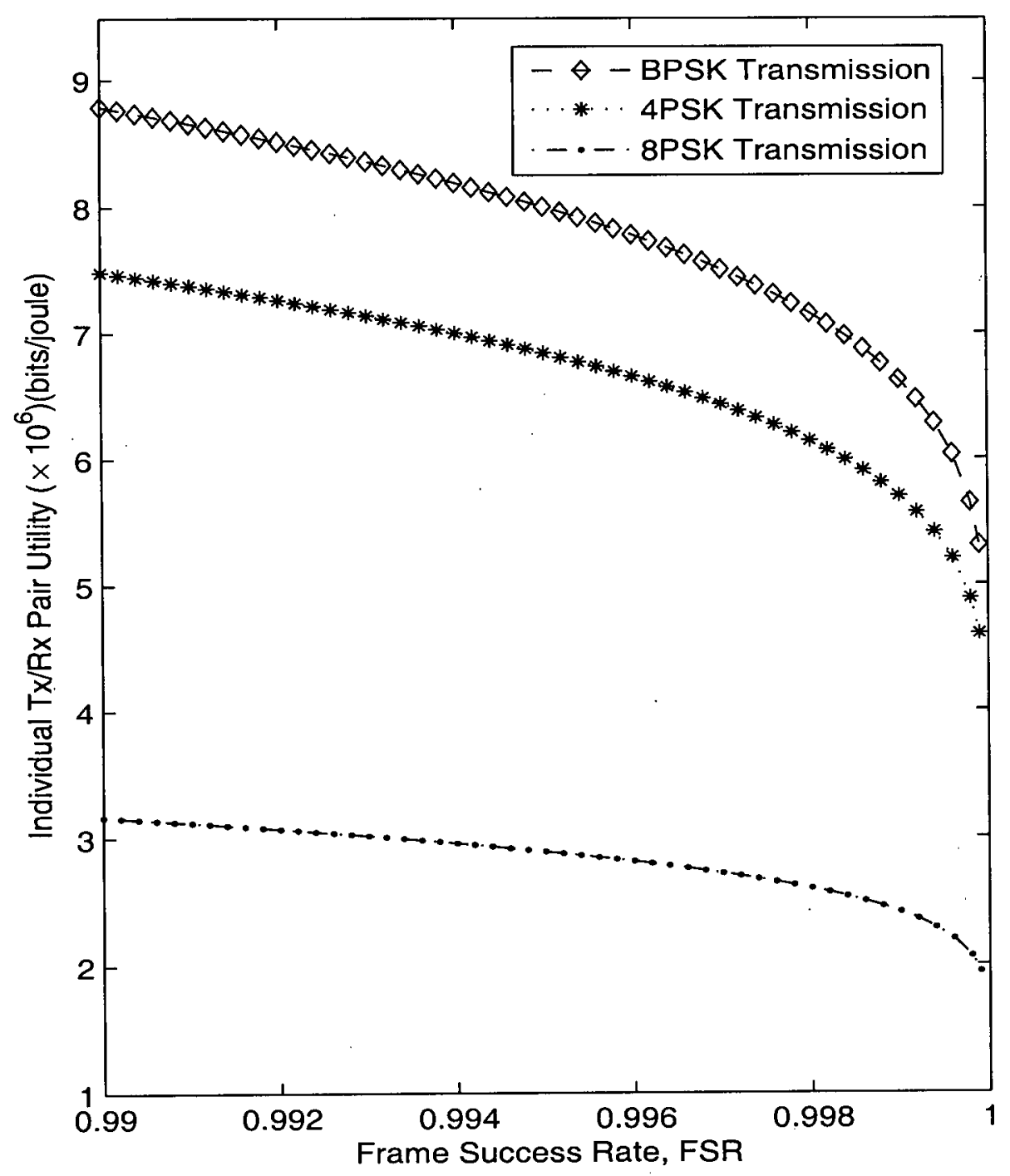

Figure 4.4: Effect of different MPSK (M=2,4,8) modulation on individual utility function. 




Figure 4.5: Effect of different MPSK $(M=16,32,64)$ modulation on individual utility function. 


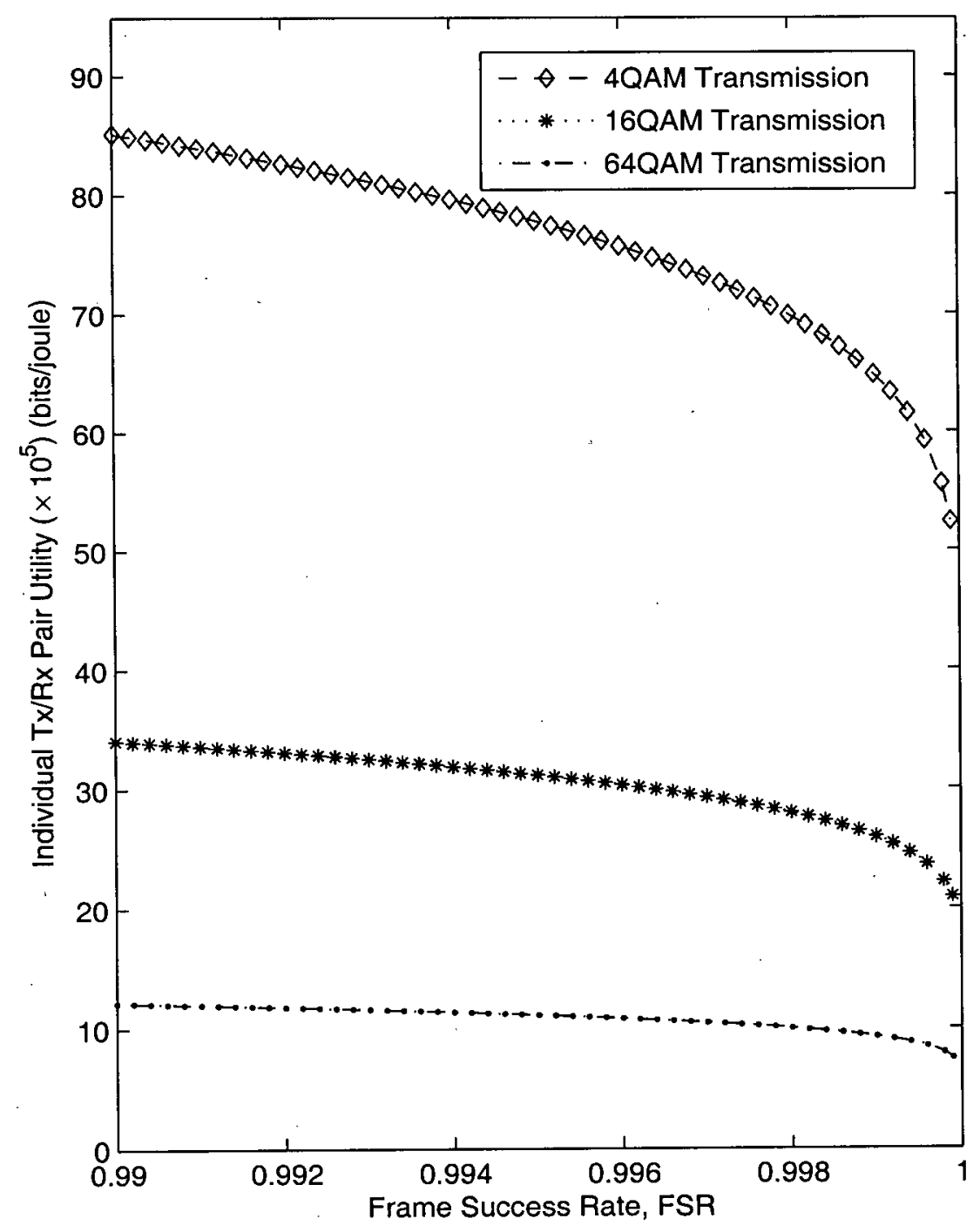

Figure 4.6: Variation of individual utility function with FSR for different MQAM. 


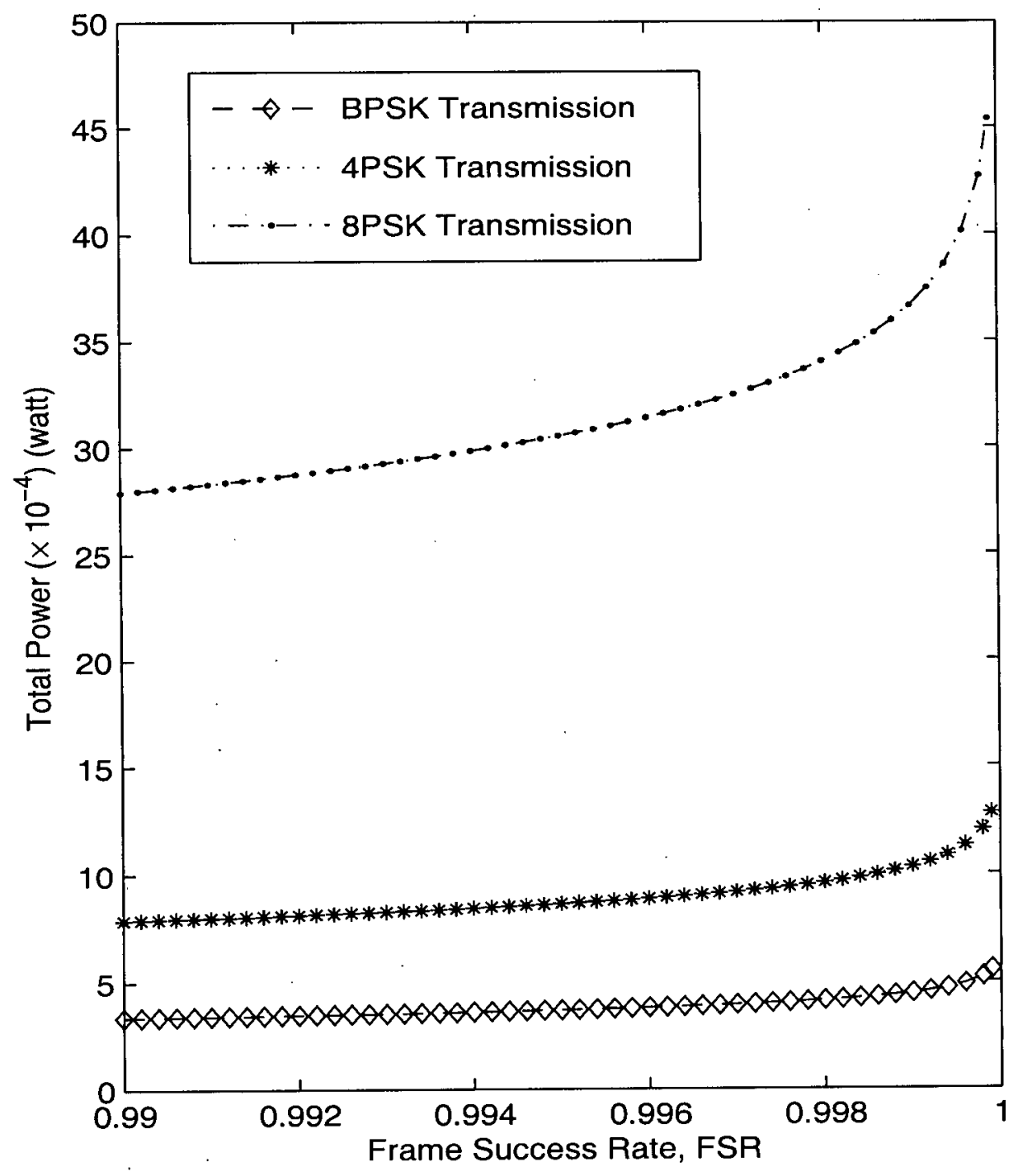

Figure 4.7: Dependence of total transmission power on FSR for different MPSK modulation $(M=2,4,8)$. 


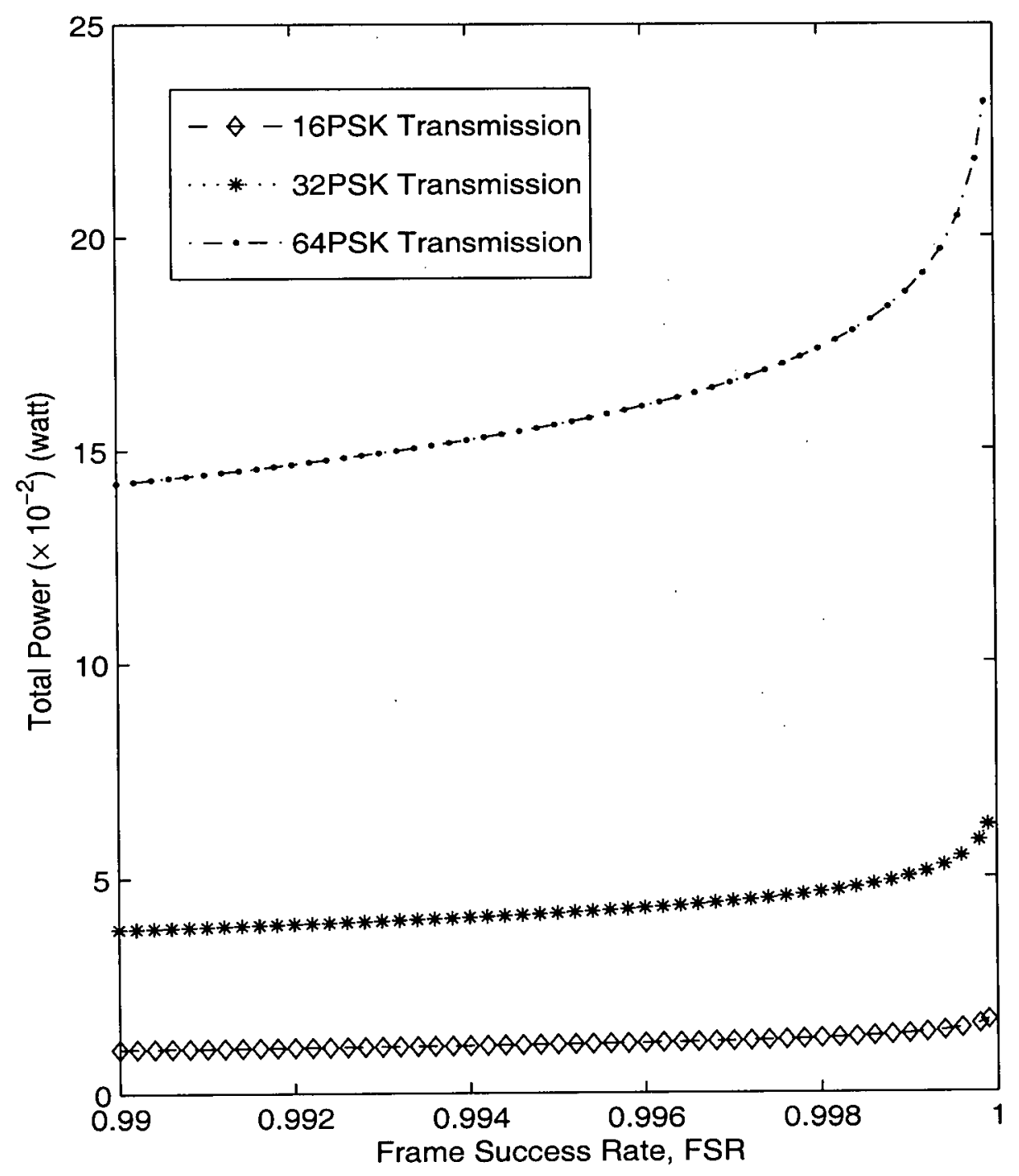

Figure 4.8: Dependence of total transmission power on FSR for different MPSK modulation $(M=16,32,64)$. 


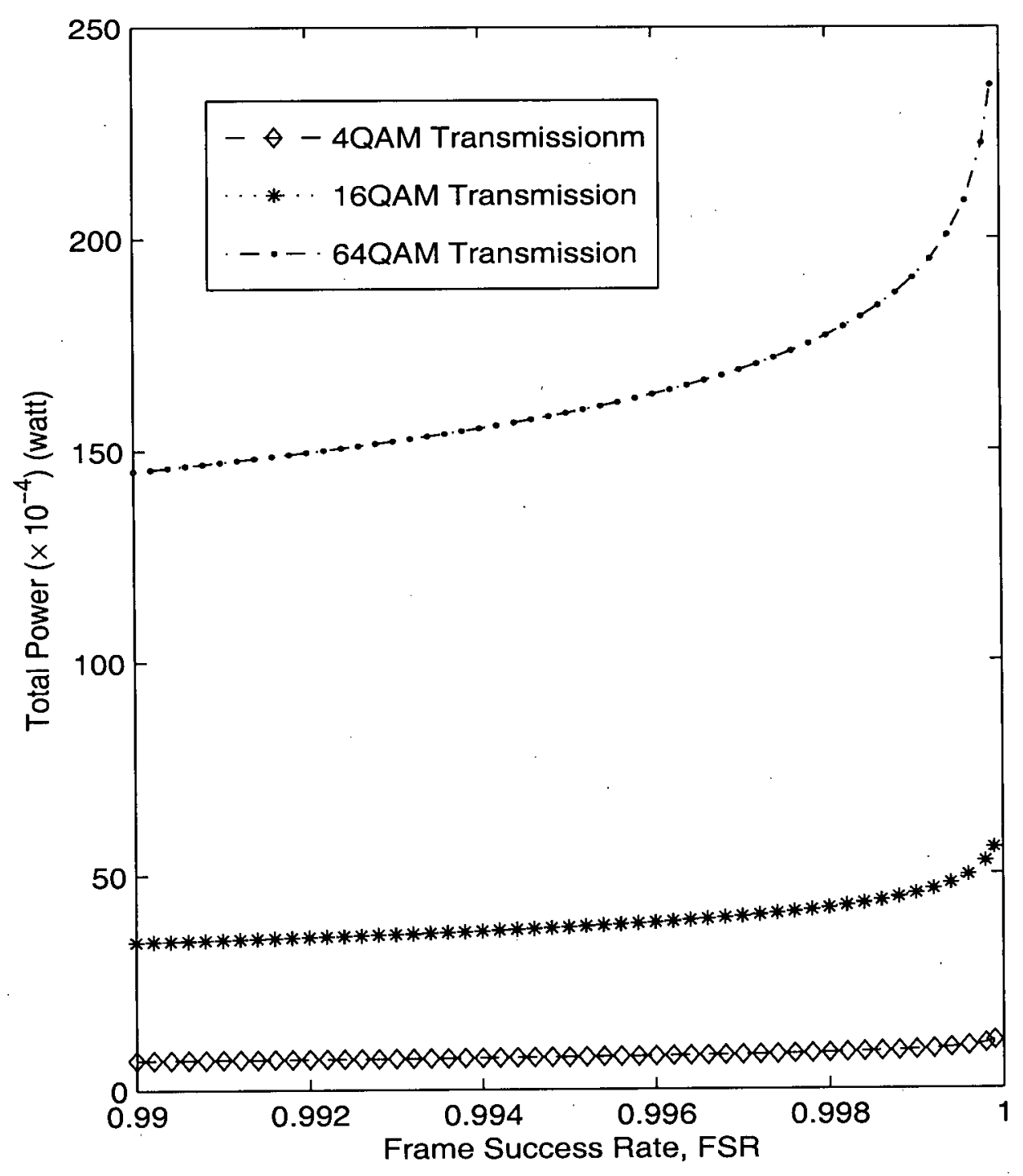

Figure 4.9: Variation of total transmission power on FSR for different MQAM. 


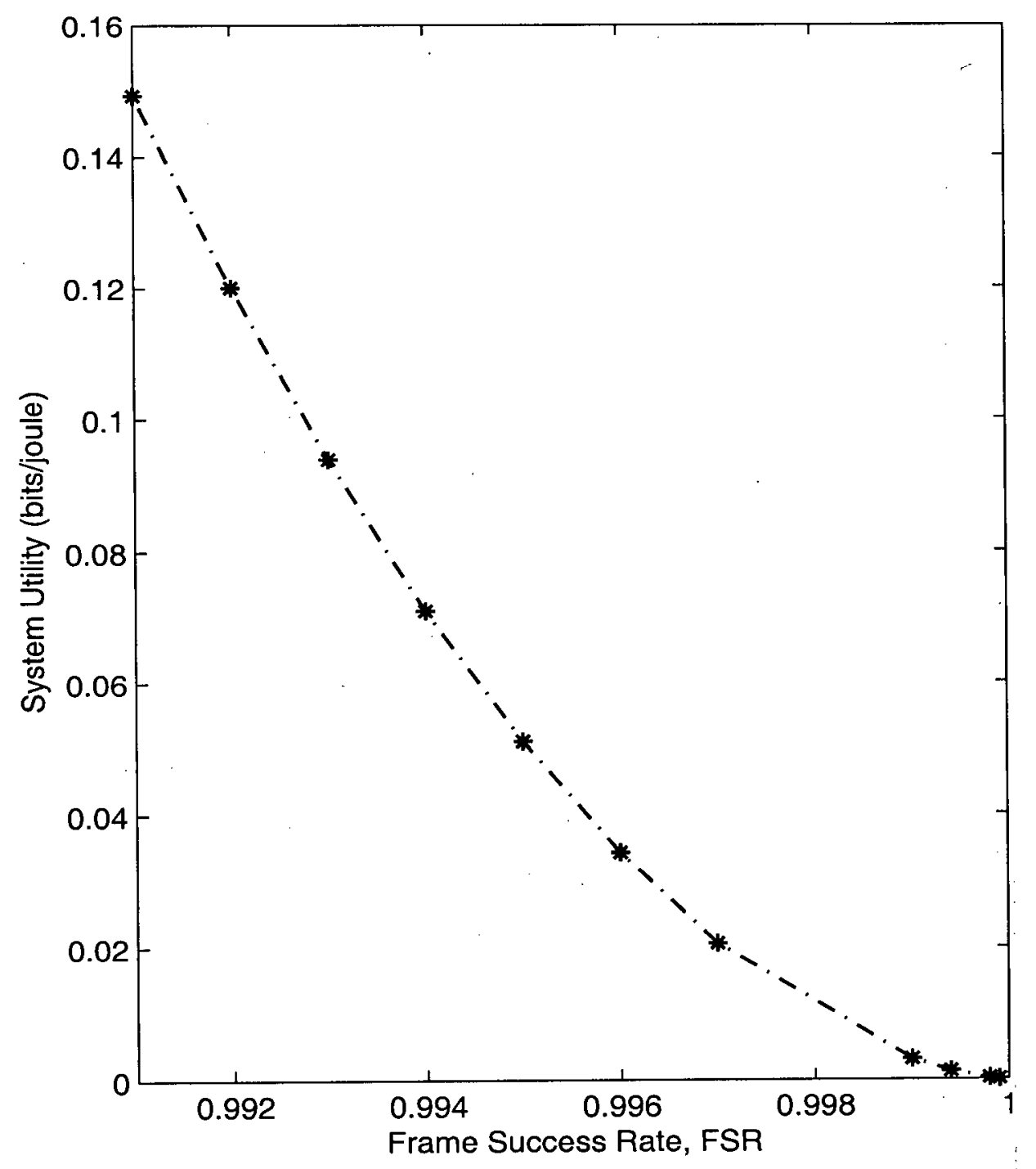

Figure 4.10: Variation of system utility with FSR. 


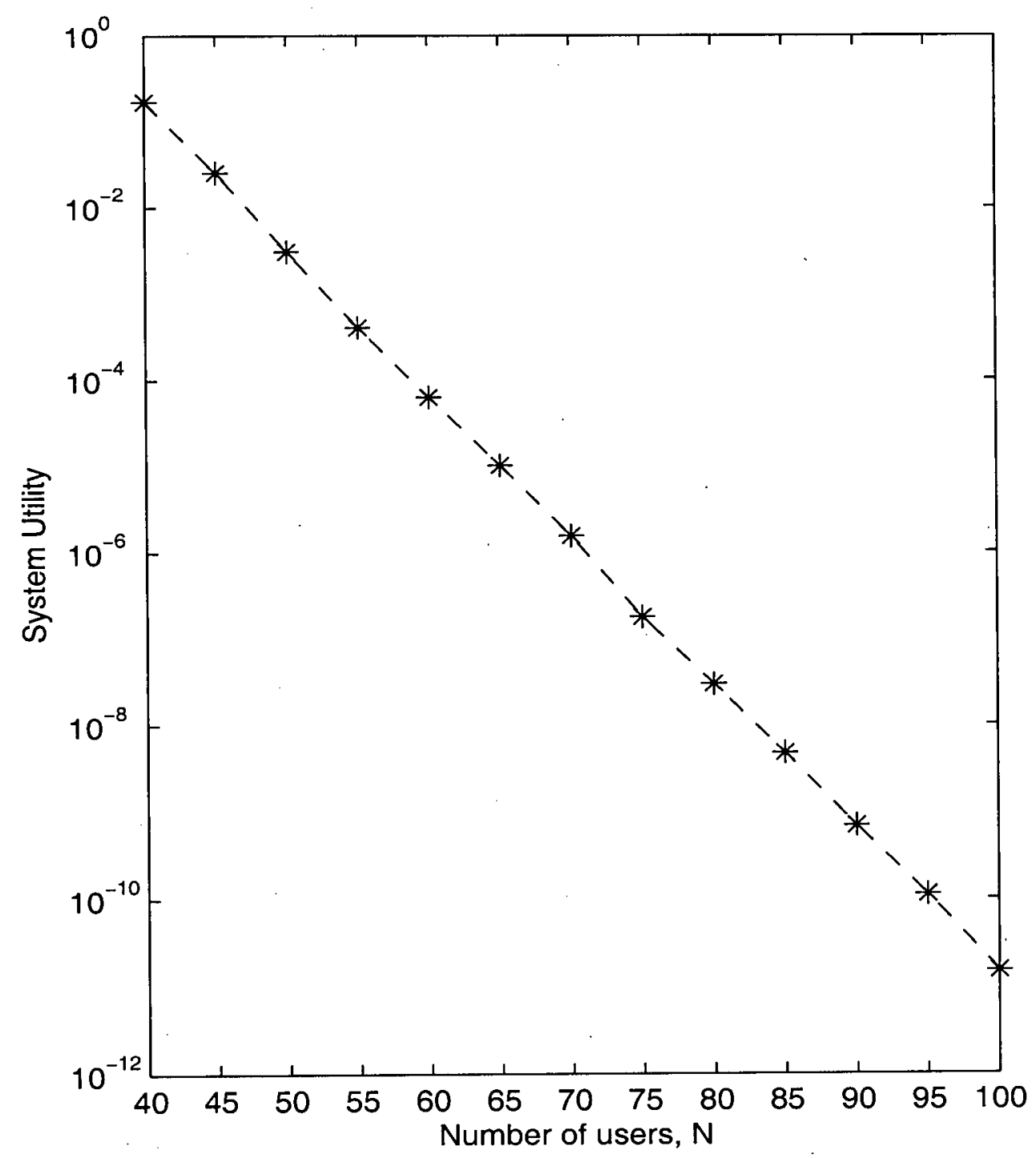

Figure 4.11: Variation of system utility with number of $\mathrm{Tx} / \mathrm{Rx}$ pairs. 


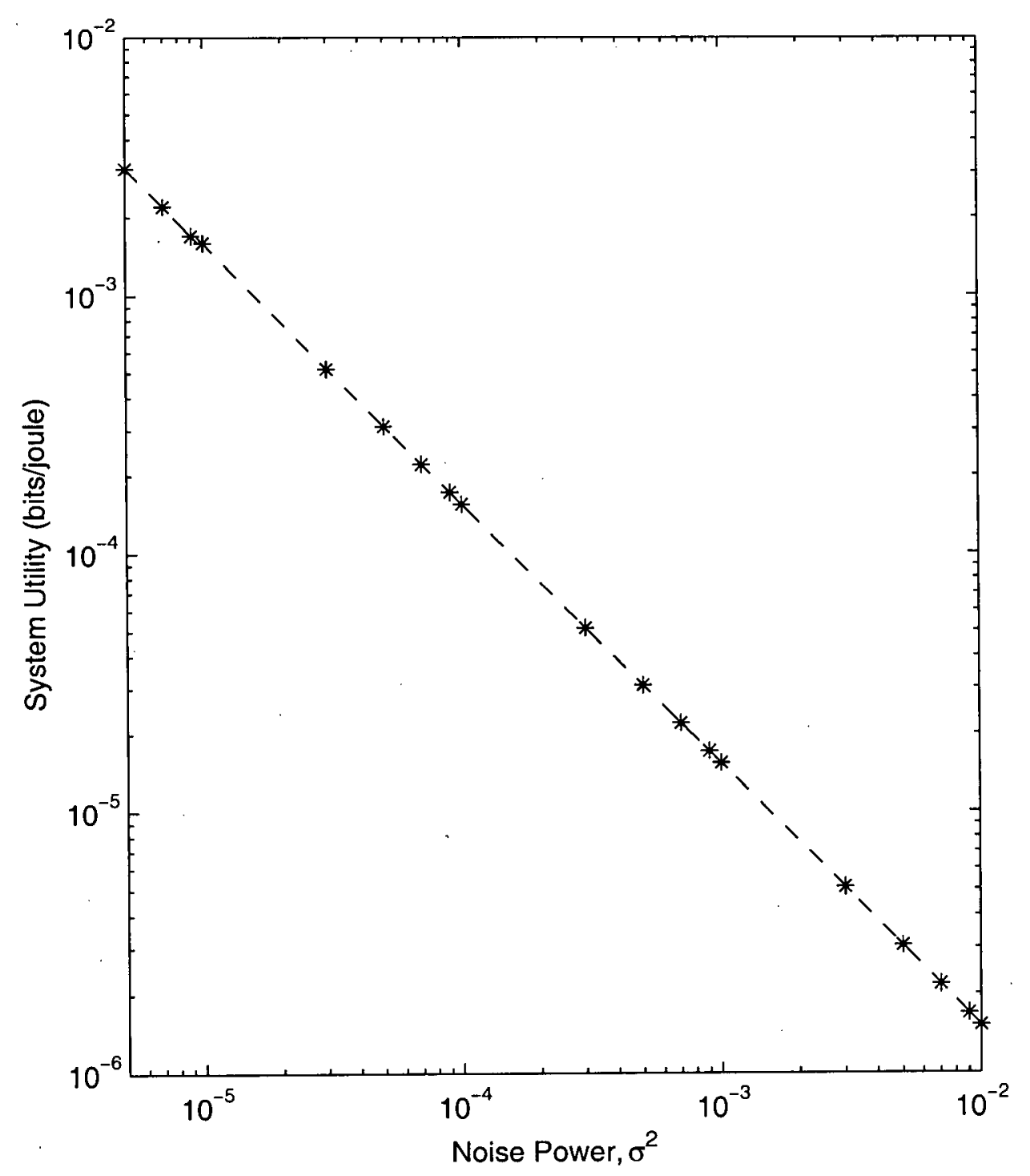

Figure 4.12: Variation of system utility with noise power. 


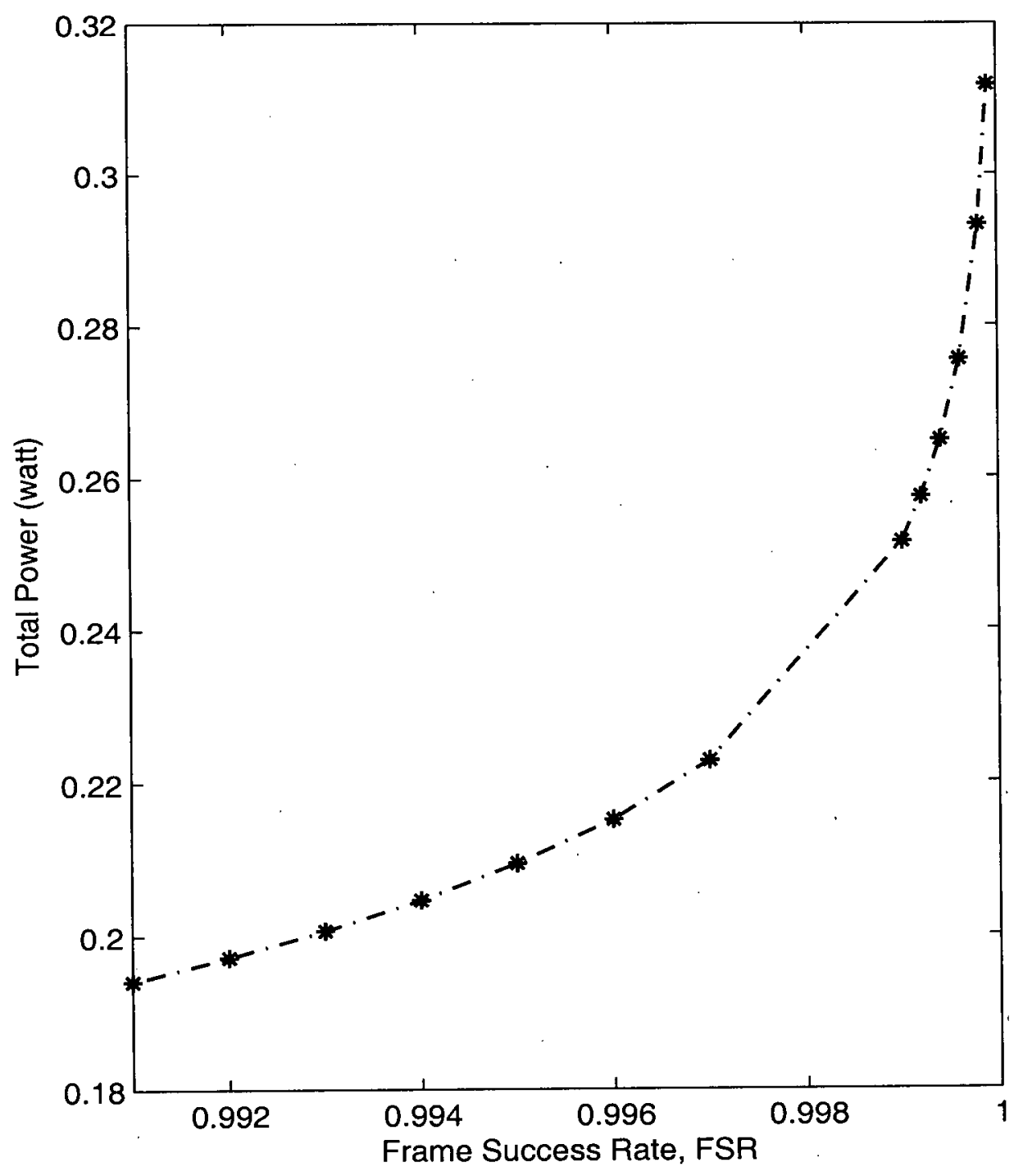

Figure 4.13: Variation of total transmitted power with FSR. 


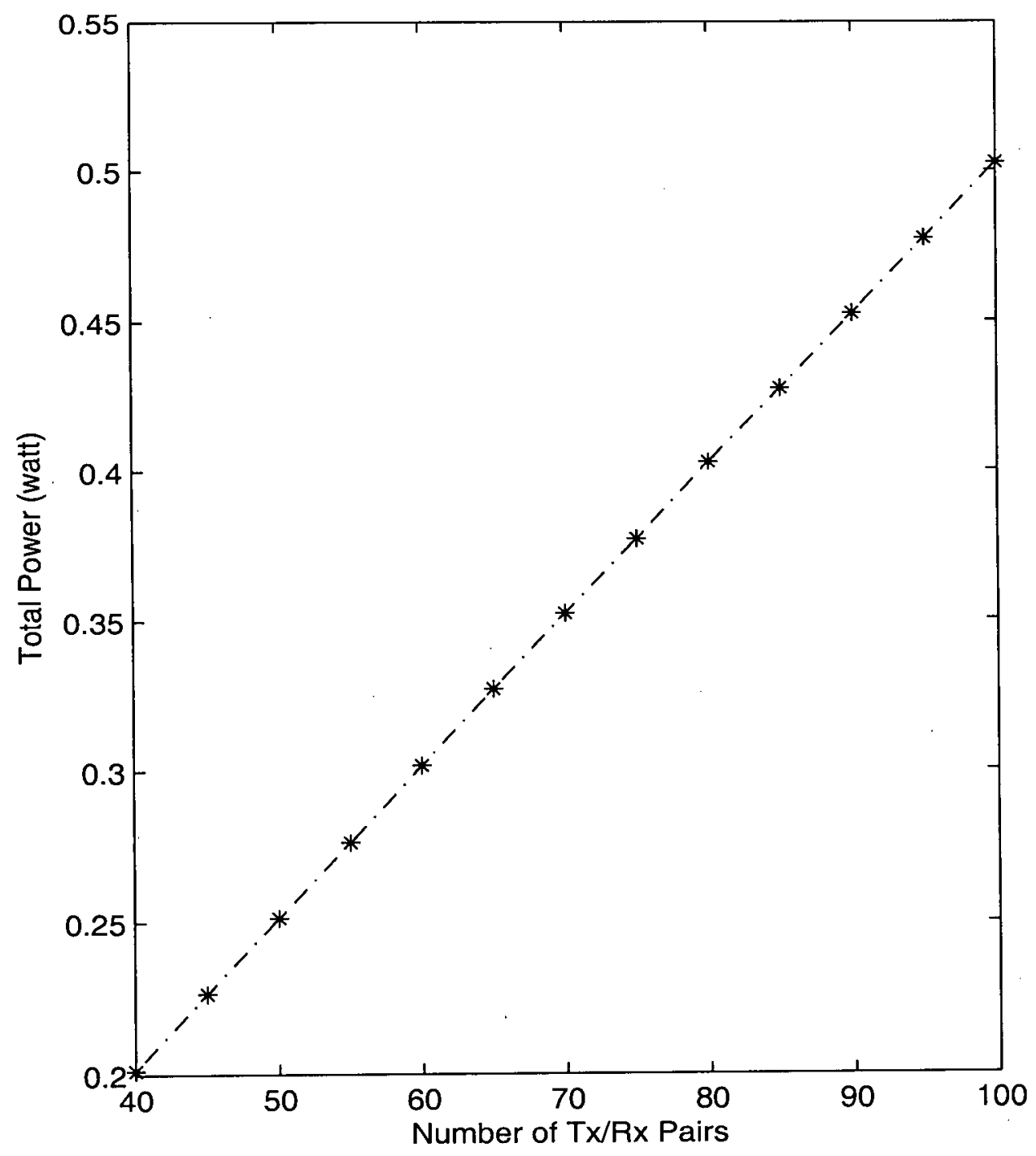

Figure 4.14: Variation of total transmitted power with number of $\mathrm{Tx} / \mathrm{Rx}$ pairs. 


\section{Chapter 5}

\section{Outage-constraint Total Power Minimization}

\subsection{Introduction}

In Chapter 3, we formulate the problem to minimize outage probability with constraint on the transmitter powers. We give an alternative formulation of this problem in this chapter. The objective of the problem is to minimize total power so that the outage probability is less than or equal to some specified value and individual power is nonnegative. We discuss the detailed formulation and solution in Section 5.2. Simulation results are given in Section 5.3.

\subsection{Problem Formulation}

In this section, we consider the problem of minimizing total transmitter power subject to outage constraints and bounds on individual powers. To minimize the total transmit power, subject to the constraint that each transmitter/receiver can attain a maximum allowed outage probability and subject to nonnegativity bound on the individual trans- 
mitter powers, we form the problem

$$
\begin{array}{cl}
\text { minimize } & f=\sum_{i=1}^{N} P_{i} \\
\text { subject to } & O_{i}<O_{i}^{\max }, i=1,2, \cdots, N \\
& P_{i} \geq 0, i=1,2, \cdots, N,
\end{array}
$$

where, $O_{i}$ is the outage probability and is given by (3.5). The number $O_{i}^{\max }$ is the bound for the outage probability for the $i^{\text {th }}$ transmitter/receiver pairs. Note that these can be the same or different for each pair, allowing different QoS to be assigned to different users. Evidently, the outage-probability constraints are the challenging ones, since $O_{i}$ is a highly nonlinear function of the powers.

The second constraints can be eliminated by letting $P_{i}=x_{i}^{2}, i=1,2, \cdots, N$. Therefore, the modified problem becomes,

$$
\begin{array}{cl}
\text { minimize } & f=\sum_{i=1}^{N} x_{i}^{2} \\
\text { subject to: } & 1-e^{-\frac{\sigma^{2} \operatorname{SINR}_{i i} x_{i}^{2}}{G_{i}}} \prod_{k \neq i} \frac{1}{1+\frac{\operatorname{SINR}^{t h} G_{i k} x_{k}^{2}}{G_{i i} x_{i}^{2}}}<O_{i}^{\max }, i=1,2, \cdots, N .
\end{array}
$$

The first constraints can be eliminated by modifying the objective function using Barrier Method. Barrier Method solves the inequality constrained problem by solving a sequence of unconstrained problems with twice differentiable objective. The most widely used barrier function is the logarithmic barrier, defined as follows,

$$
\phi(x)=-\sum_{i=1}^{N} \log \left(-f_{i}(x)\right)
$$

with dom $\phi=\left\{x \mid f_{i}(x)<0, i=1, \ldots, N\right\} . \phi$ is called the logarithmic barrier function; it is twice differentiable and convex. Its domain is the set of strictly feasible points and it tends to infinity as $x$ approaches the boundary of $\operatorname{dom} \phi$.

Therefore in our problem we can eliminate the constraints on the outage by modifying the objective function as follows

$$
\text { minimize } f=\sum_{i=1}^{N} x_{i}^{2}-\sum_{i=1}^{N} \mu_{i} \log b_{i}
$$


where, $b_{i}$ is given by,

$$
b_{i}=\left(O_{i}^{m a x}-1+e^{-\frac{\sigma^{2} \operatorname{sINR} t h}{G_{i i} x_{i}^{2}}} \prod_{k \neq i} \frac{G_{i i} x_{i}^{2}}{G_{i i} x_{i}^{2}+\operatorname{SINR}^{t h} G_{i k} x_{k}^{2}}\right)>0, i=1,2, \cdots, N .
$$

Here, $\mu_{i}, i=1,2, \cdots, N$ are strictly positive numbers and called barrier parameters.

The term $-\sum_{i=1}^{N} \mu_{i} \log b_{i}$ is logarithmic barrier function and starting with an initial $b_{0}$ which is strictly inside the feasible region, we obtain a barrier that prevents any component $b_{i}$ from becoming zero. The effect of the barrier function on the original problem depends largely on the magnitude of $\mu_{i}$. Solving the equation (5.8) for a series values of $\mu_{i}$, a series solutions of the objective function are obtained that converge to the optimal value as $\mu_{i}$ approaches to zero.

Now the problem (5.7) becomes an unconstrained optimization problem and the general algorithm for solving the problem is given in Table 5.1. Note that when the number of users is small, the gradient can be found analytically. But, for large number of user, the gradient of the problem can be determined numerically.

\subsubsection{Example: Two Users Case}

To show how the formulation converged to the optimal point with the given bounds, in this section, we consider a simple example with two users. The analytical expression for objective function is given by,

minimize $f=x_{1}^{2}+x_{2}^{2}-\mu_{1} \log \left(O_{\text {max }}-\frac{\operatorname{SINR}^{\text {th }} G_{12} x_{2}^{2}}{D_{12}}\right)-\mu_{2} \log \left(O_{\text {max }}-\frac{\operatorname{SINR}^{\text {th }} G_{21} x_{1}^{2}}{D_{21}}\right)$

where, $D_{i j}=G_{i i} x_{i}^{2}+\operatorname{SINR}^{t h} G_{i j} x_{j}^{2} ; i, j=1,2$. The outage bound of two users are taken equal, i.e., $O_{1}^{\max }=O_{2}^{\max }=O_{\max }$, and the ambient white-noise power is considered insignificant compared to interference power. The analytical expression for the gradient vector is given by,

$$
\overrightarrow{g(x)}=\nabla \vec{f}=\left[\begin{array}{ll}
\frac{\partial f}{\partial x_{1}} & \frac{\partial f}{\partial x_{2}}
\end{array}\right]^{T}
$$




\section{Algorithm 4}

Step 1: Find an initial point $\overrightarrow{x_{0}}$ that satisfies all the constraint given in (5.8). Input tolerance $\epsilon$ and the multiplier $\mu_{i}=1, i=1,2, \cdots, N$. Set $k=0$ and $\overrightarrow{x_{k}}=\overrightarrow{x_{0}}$.

Step 2: Find the optimum value of the vector $\overrightarrow{x_{k+1}}$ that are within feasible region using either DFP or BFGS algorithm discussed in Appendix A.2 or Appendix A.3 respectively.

Step 3: If $\left\|\left(\overrightarrow{x_{k+1}}-\overrightarrow{x_{k}}\right)\right\|<\epsilon$, then output $\overrightarrow{x^{*}}=\overrightarrow{x_{k+1}}$ and $f\left(x^{*}\right)=\sum_{i=1}^{N} x_{i}^{2}$.

Step 4: $\quad$ Set $k=k+1, \overrightarrow{x_{k}}=\overrightarrow{x_{k+1}}$ and $\mu_{i}=0.1 \mu_{i}, \quad i=$ $1,2, \cdots, N$ and repeat from Step 2 .

Table 5.1: Algorithm for solving outage-constraint total power minimization problem

where,

$\frac{\partial f}{\partial x_{1}}=2 x_{1}-\frac{2 \mu_{1} \operatorname{SINR}^{\text {th }} G_{11} G_{12} x_{1} x_{2}^{2}}{D_{12}\left(O_{\max } D_{12}-\operatorname{SINR}^{t h} G_{12} x_{2}^{2}\right)}+\frac{2 \mu_{2}\left(x_{1} \operatorname{SINR}^{\text {th }} G_{21} D_{21}-\operatorname{SINR}^{\text {th }} G_{21}^{2} x_{1}^{3}\right)}{D_{21}\left(O_{\max } D_{21}-\operatorname{SINR}^{t h} G_{21} x_{1}^{2}\right)}$

and

$\frac{\partial f}{\partial x_{2}}=2 x_{2}-\frac{2 \mu_{2} \operatorname{SINR}^{t h} G_{21} G_{22} x_{1}^{2} x_{2}}{D_{21}\left(O_{\max } D_{21}-\operatorname{SINR}^{t h} G_{21} x_{1}^{2}\right)}+\frac{2 \mu_{1}\left(x_{2} \operatorname{SINR}^{\text {th }} G_{12} D_{12}-\operatorname{SINR}^{\text {th }} G_{12}^{2} x_{2}^{3}\right)}{D_{12}\left(O_{\max } D_{12}-\operatorname{SINR}^{\text {th }} G_{12} x_{2}^{2}\right)}$

We give some numerical results for this example in the next section.

\subsection{Numerical Results}

For the numerical results, we take all the gains $G_{i i}, i=1,2$ to be one, and we generate the cross gains $G_{i j}, i \neq j$ as independent random variables uniformly distrib- 
Table 5.2: Numerical results for example in Section 5.2.1

\begin{tabular}{|c|c|c|c|}
\hline Initial Values: $\vec{\mu}, \overrightarrow{x_{0}}$ & $\vec{P}=\left[\begin{array}{ll}P_{1} & P_{2}\end{array}\right]^{T}$ & $f_{\text {original }}$ & $k$ \\
\hline$\left[\begin{array}{ll}1 & 1\end{array}\right]^{T},\left[\begin{array}{lll}0.4 & 2.0\end{array}\right]^{T}$ & $10^{-8} \times\left[\begin{array}{lll}7.995 & 3.94\end{array}\right]^{T}$ & $1.1935 \times 10^{-7}$ & 14 \\
\hline $10^{-1} \times\left[\begin{array}{ll}1 & 1\end{array}\right]^{T}, 10^{-4} \times\left[\begin{array}{lll}2.827 & 1.985\end{array}\right]^{T}$ & $10^{-8} \times[7.9923 .94]^{T}$ & $1.1932 \times 10^{-7}$ & 1 \\
\hline $10^{-2} \times\left[\begin{array}{ll}1 & 1\end{array}\right]^{T}, 10^{-4} \times\left[\begin{array}{lll}2.827 & 1.985\end{array}\right]^{T}$ & $10^{-8} \times\left[\begin{array}{lll}7.989 & 3.941\end{array}\right]^{T}$ & $1.1930 \times 10^{-7}$ & 1 \\
\hline $10^{-3} \times\left[\begin{array}{ll}1 & 1\end{array}\right]^{T}, 10^{-4} \times\left[\begin{array}{lll}2.827 & 1.985\end{array}\right]^{T}$ & $10^{-8} \times[7.7243 .851]^{T}$ & $1.1575 \times 10^{-7}$ & 1 \\
\hline
\end{tabular}

uted between zero and 0.001 . The values for other parameters are: $O_{\max }=0.1$ and $\mathrm{SINR}^{\text {th }}=3$. We show the mesh plot of feasible region for the function in figure 5.1. The feasible region is defined as the set of all points that satisfy the constraints. The optimum point must be located in the feasible region. The contour for the function in the feasible region is given in Fig. 5.2. Contour plot is a graphical method used to find the minimum and the maximum of the objective function. It is defined as a set of points in the $\left(x_{1}, x_{2}\right)$ plane for which $f\left(x_{1}, x_{2}\right)$ is constant. And so a contour plot, like a topographical map of a specific region, will reveal readily the peaks and valleys of the function. In our example we show the contour plot for function value $0.1,8,12,16$, $20,24,28,32$. But the graphical method is limited usefulness since it is not possible to plot the graph if the number of variables exceed two. We use DFP and BFGS method to find the solutions which is more practical since the number of variables does not put a limitation in this case.

We start the algorithms for initial values of $x_{0}=\left[\begin{array}{ll}0.42 & 2\end{array}\right]$ and $\mu_{i}=\left[\begin{array}{ll}1 & 1\end{array}\right], i=1,2$. The results using DFP algorithm for $\mu_{i}$ upto $10^{-3}$ is given in Table 5.2. 


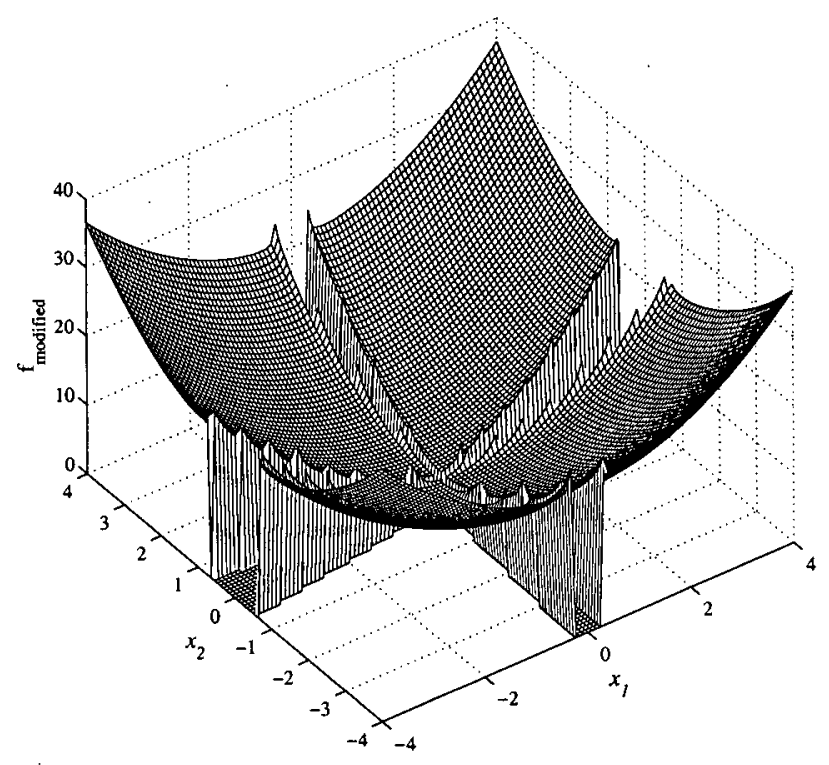

Figure 5.1: Mesh plot of modified objective function in the feasible region



Figure 5.2: Contour plot of the modified objective function in the feasible region 


\section{Chapter 6}

\section{Conclusions and Future Directions}

This final chapter summarizes the main contributions of the thesis, and discusses the advantages, limitations and applications of the proposed algorithms. Further works to extend the results of this thesis is also presented. A summary of result is presented in Section 6.1, whereas interesting and important future research directions are suggested in Section 6.2.

\subsection{Summary}

The inherent limitations of cellular wireless systems and the strong demand for highperformance personal communications require efficient management of radio resources. Power control is a technique that not only improves spectral efficiency but also can be used as a platform for resource management. Among various resource management methods, efficient power control is important for reducing radiation levels and extending battery lives of mobile devices. Furthermore, it is crucial for interference-limited wireless networks such as CDMA, where optimal control of transmit powers can lead to effective interference and hence outage minimization, and increased capacity of network.

We have demonstrated both centralized and distributed radio resource manage- 
ment for multimedia wireless networks. For voice communications, we have presented outage-based optimal power control techniques in interference-limited environments with Rayleigh fading signal and interferences. We have discussed two different formulations for outage-based power control. Whereas in first formulation outage is minimized with constraint on transmitter powers, in second formulation total power is minimized with bounds on outage probability. Because of different nature of traffic and QoS requirements, outage-based power control schemes is not suitable for data. We have considered utility-based power control schemes for data services in the same Rayleigh faded interference-limited environments. The utility is maximized from the viewpoint of user and network respectively. We have introduced simple unconstrained based formulations and efficient solution techniques.

The benefit of the outage-probability minimizing/utility-maximizing method of allocating power is that it allows power allocation to be done on the far longer time scale than Rayleigh fading time scale, which is often log normal shadowing time scale. Since power is not updated with changing fading state, this method consumes less signal processing costs and it is more practical method of allocating power for fast fading channels. The disadvantage of this method is a positive probability of fading-induced outage. Of course, this disadvantage is also present in a power-allocation method that attempts to track fading state: for some fading states, allocating power to guarantee reception for all transmitter/receiver pairs is impossible. Of course, outage probability can be reduced by allocating power in such a way that each mobile has an extra margin of SINR. Proposed outage-based or utility-based power control algorithms can be implemented, for example in adhoc wireless networks, at the log-normal shadowing time scale rather than the Rayleigh fading time-scale. 


\subsection{Future Works}

There are many open problems in the area of power control. A primary problem is power control in stochastic system, where the randomness arises in wireless channel, mobility, and data source.

In our work, we have presented uplink/downlink power control algorithms in the presence of Rayleigh fading. Nakagami-m fading channels with different fading channel parameters such as correlation coefficient and rate of fading, channel variations, etc. can be considered in future works.

In our work, we have considered both the user-centric and network-centric case separately. User-centric and network-centric RRM schemes are motivated by different interests, and hence, ought to result in dissimilar resource allocations. User-centric schemes tend to distributed QoS more evenly to users than network-centric ones. On the other hand, network-centric measurement reflects the total revenue derived by the usage of network interests that not to appear in the solutions of user-centric management. Therefore, a joint network-centric and user-centric consideration should produce an interesting solution, and be worth exploring a research topic.

Utility-based power control provides a promising framework for distributed power control of multimedia cellular wireless systems. But it is still not well-developed and several issues remain to be studied. How to translate different QoS requirements into utility and cost functions that lead to a solvable power-control problem, how to achieve system optimality, and how to relate a cost term to practical pricing schemes are all topics requiring further research.

Finally, we conclude that the performance of future wireless networks will depend on the design of dynamic channel allocation, traffic scheduling, adaptive antenna, and adaptive modulation/coding for link adaptation. So far these issues are considered separately. With the proposed algorithm as a basis, we have to take into account all the issues jointly to achieve high spectral efficiency and capacity in practical networks. 


\section{Bibliography}

[1] Piplu R. Paul, Ashok K. Karmokar, and Vijay K. Bhargava, "Optimal power control for network-centric and user-centric wireless networks in interference-limited fading channels," in Proc. of IEEE Canadian Conference on Electrical and Computer Engineering (CCECE'05), Saskatoon, Saskatchewan, Canada, May 1-4, 2005 .

[2] J. Walrand, and P. Varaiya, High-Performance Networks, 2nd ed., Morgan Kaufmann Publishers: San Francisco, 2000.

[3] R. Prasad, Universal Wireless Personal Communications. Artech House Publishers: Boston, MA, 1998.

[4] M. Xiao, N. B. Shroff, and E. K. P. Chong "Resource management in powercontrolled cellular wireless systems," Wireless Commun. Mob. Comput., vol. 1, pp. 185-199, 2001.

[5] S. Verdu, "Wireless bandwidth in the making" IEEE Commun. Mag., vol. 38, pp. 53-58, Jul. 2000

[6] C. U. Saraydar, N. B. Mandayam, and D. J. Goodman, "Efficient power control via pricing in wireless data networks," IEEE Trans. Commun., vol. 50, pp. 291-303, Feb. 2002.

[7] T. S. Rappaport, Wireless Communications:Principles and Practice. New Jersey: Prentice Hall Inc., 2002. 
[8] 3GPP TS 45.008. V6.4.0, "Radio access network; radio subsystem link control," Sep. 2003.

[9] A. Viterbi, CDMA: Principles of spread spectrum communication. Prentice Hall PTR: NJ, 1995.

[10] J. Zander, "Performance of optimum transmitter power control in cellular radio systems," IEEE Trans. Veh. Technol., vol. 41, pp. 57-62, Feb. 1992.

[11] F. Berggren, S-L. Kim, R. Jantti, and J. Zander, "Joint power control and intracell scheduling of DS-CDMA nonreal time data," IEEE J. Select. Areas Commun., vol. 19 , pp. 1860-1870, Oct. 2001.

[12] J. Zander, "Trends in resource management future wireless networks," in Future Telecommunications Forum, FTF 99, Beijing, China, December 1999.

[13] M. Xiao, N. B. Shroff, and E. K. P. Chong, 'A utility-based power control scheme in wireless cellular systems," IEEE/ACM Trans. Networking, vol. 11, pp. 1-8, Apr. 2003.

[14] A. Kajacks, V. Batkauskas, and A. Medeisis, "Individual QoS rating for voice services in cellular networks," IEEE Commun. Mag., vol. 42, pp. 88-93, Jun. 2004.

[15] R. D. Yates, "A framework for uplink power control for cellular radio systems," IEEE J. Select. Areas Commun., vol. 13, pp. 1341-1347, Sep. 1995.

[16] Z. J. Haas, J. H. Witers, and D. S. Johnson, 'Simulation results of the capacity of cellular systems," IEEE Trans. Veh. Technol., vol. 46, pp. 805-817, Nov. 1997.

[17] J. F. Whitehead, "Signal-level-based dynamic power control for co-channel interference management," in Proc. IEEE Veh. Techn. Conf, Secaucus, NJ, May 1993, pp. 499-502. 
[18] G. J. Foschini, and Z. Miljanic, "A simple distributed autonomous power control algorithm and its convergence," IEEE Trans. Veh. Technol., vol. 42, pp. 641-646, Nov. 1993.

[19] S. A. Grandhi, R. Vijayan, and D. J. Goodman, "Distributed power control in cellular radio systems," IEEE Trans. Commun., vol. 42, pp. 226-228, Apr. 1994.

[20] J. Zander, "Distributed cochannel interference control in cellular radio systems," IEEE Trans. Veh. Technol., vol. 41, pp. 305-311, Aug. 1992.

[21] S. Ariyavisitakul, "SIR based power control in a CDMA system," in Proc. GLOBECOM'92, Dec. 1992, pp. 868-873.

[22] Y. Nagata, and Y. Akaiwa, "Analysis for spectrum efficiency in single cell trunked and cellular mobile radio," IEEE Trans. Veh. Technol., vol. 35, pp. 100-113, Aug. 1987.

[23] R. Muammar, and S. C. Gupta, "Cochannel interference in high capacity mobile radio systems," IEEE Trans. Commun., vol. 30, pp. 1973-1978, Aug. 1982.

[24] K. Daikoku, and H. Ohdate,, "Optimal channel reuse in cellular land mobile radio systems.," IEEE Trans. Veh. Technol., vol. 32, pp. 217-224, Aug. 1983.

[25] R. Prasad, A. Kegel, and J. C. Arnbak, "Analysis of system performance of highcapacity mobile radio," in Proc. IEEE VTC'89, San Fransisco, CA, May 1989, pp. 306-310.

[26] R. C. French, "The effect of fading and shadowing on channel re-use in mobile radio," IEEE Trans. Veh. Technol., vol. 28, pp. 171-181, Aug. 1979.

[27] J. M. Aein, "Power balancing in systems employing frequency reuse," COMSAT Tech. Rev., vol. 3, no. 2, Fall 1973. 
[28] R. W. Nettleton, “ Traffic theory and interference management for a spread spectrum cellular radio system," Proc. ICC'80, Seattle, WA, 1980.

[29] R. W. Nettleton, and H. Alavi, "Downstream power control for spread spectrum cellular mobile radio system," Proc. Globecom'82, Miami, FL, 1982.

[30] S. A. Grandhi, R. Vijayan, D. J. Goodman, and J. Zander, "Centralized power control in cellular radio systems," IEEE Trans. Veh. Technol., vol. 42, pp. 466-468, Nov. 1993.

[31] G. Femenias, F. J. Perze-Briceno, A. Gelonch, and I. Furio, "Transmitter power control for DS/CDMA cellular mobile radio networks," in proc. IEEE PIMRC'95, 1995, vol. 1, pp. 46-50.

[32] M. Zorzi, "Power control and diversity in mobile radio cellular systems in the presence of Rician fading and log-normal shadowing," IEEE Trans. Veh. Technol., vol. 45, pp. 373-382, May. 1996.

[33] A. A. Abu-Dayya, and N. C. Beaulieu, "Outage probabilities of cellular mobile radio systems with multiple nakagami interferers," IEEE Trans. Veh. Technol., vol. 40, pp. 757-767, Nov. 1992.

[34] S. Ulukus, and R. D. Yates, "Stochastic power control for cellular radio systems," IEEE Trans. Commun., vol. 46, pp. 784-798, Jun. 1998.

[35] S. C. Chen, N. Bamboos, and G.J. Pottie, "Admission control schemes for wireless communication networks with adjustable transmitter powers," in Proc. IEEE INFOCOM'94, Toronto, CANADA, Jun. 1994, pp.21-28.

[36] D. Mitra, "An asynchronouus distributed algorithm for power control in cellular radio systems," in Proc. 4th Winlab Workshop Third Generation Wireless Information Network, New Brunswick, NJ, Oct. 1993, pp. 249-257 
[37] D.C. Cox, "Cochannel interference considerations in frequency re-use smallcoverage area radio systems," IEEE Trans. Commun., vol. 30, pp. 135-142, Jan. 1982.

[38] Yu-Shuan Yeh, and S. C. Schawartz, "Outage Probability in mobile telephony due to multiple log-normal interferers," IEEE Trans. Commun., vol. 4, pp. 380-388, Apr. 1984.

[39] G. K. Chan, "Design and analysis of a land mobile cellular system under the effects of interferers," Ph. D. dissertation, Carleton University, Canada, 1984.

[40] K. Sowerby, and A. Williamson, "Outage probability calculations for mobile radio systems with multiple interferers," Electron. lett., vol. 24, pp. 2073-2075, Aug. 1988.

[41] J. M. G. Linnartz, "Exact analysis of the outage probability in multiple-user mobile radio," IEEE Trans. Commun., vol. 1, pp. 1121-1124, Jan. 1992.

[42] K. W. Sowerby, and A. G. Williamson, "Outage probabilities in mobile radio systems suffering cochannel interference," IEEE J. Select. Areas Commun., vol. 10, pp. 516-522, Apr.1992.

[43] Y.-W. Leung, "Power controls in cellular networks subject to measurement error," IEEE Trans. Commun., vol. 44, pp. 772-775, Jul. 1996.

[44] K. K. Leung, "Power control by interference prediction for broadband wireless packet networks," IEEE Trans. Wireless Commun., vol. 1, pp. 256-265, Apr.2002.

[45] K. P. Tsoukatos, "Power control in a mobility environment" in Proc. IEEE VTC'97, 1997, pp. 740-744.

[46] Q. Wu, "Performance of optimum transmitter power control in CDMA cellular mobile systems," IEEE Trans. Veh. Technol., vol. 48, pp. 571-575, Mar. 1999. 
[47] Weichen Ye, and A. M. Haimovich, "Outage probability of cellular CDMA systems with space diversity, Rayleigh fading, and power control error," IEEE Commun. Lett., vol. 2, pp. 220-222, Aug. 1998.

[48] S. Kandukuri, and S. Boyd, "Optimal power control in interference-limited fading wireless channels with outage-probability specifications," IEEE Trans. Wireless Commun., vol. 1, pp. 46-55, Jan. 2002.

[49] J. Papandriopoulos, J. Evans, , and S. Dey, "Iterative power control and multiuser detection with outage probability constraints," in Proc. of ICC'03, Anchorage, Alaska, pp. 2509-2513, May 2003.

[50] H. Ji and C. -Y. Huang, "Non-cooperative uplink power contron in cellular radio systems," Wireless Networks, vol. 4, pp. 233-240, Mar. 1998.

[51] D. J. Goodman, and N. B. Mandayam, "Power control for wireless data," IEEE Personal Commun. Mag., pp. 48-54, Apr. 2000.

[52] C. Saraydar, N. B. Madayam, and D. J. Goodman, "Pareto efficiency of pricing based power control in wireless data networks," in Proc. IEEE WCNC'99, 1999, pp.21-24.

[53] P. Liu, M. L. Honig, and S. Jordan, "Forward-link CDMA resource allocation based on pricing", in Proc. IEEE WCNC'00, 2000, pp. 1410-1414.

[54] P. Marbach, and R. Berry, "Downlink resource allocation and pricing for wireless networks," in Proc. Infocom' 02, vol. 3, 2002, pp. 1470-1479.

[55] D. Mitra, and J. Morrison, "A distributed power control algorithm for bursty transmission on cellular, spread spectrum wireless systems," Wireless Information Networks, Holtzman J (ed). Kluwer:1996; 201-212

[56] K. K. Leung, "A Kalman filter method for power control in broadband wireless networks," in Proc. INFOCOM'99, 1999, pp. 948-956. 
[57] N. Bambos, and S. Kandukuri, "Power controlled multiple access in wireless communication networks", in Proc. of IEEE, IFOCOM, Tel Aviv, Israel, 2000.

[58] J. M. Rulnick, and N. Bambos, "Mobile power management for wireless communication networks", IEEE Trans. Wireless Networks, 1997, pp. 3-14

[59] J. M. Rulnick, and N. Bambos, "Power-induced time division on asynchronous channels", IEEE Trans. Wireless Networks, 1999, pp. 71-80

[60] D. S. C. Tse, and S. V. Hanly,"Multiaccess fading channels-part I: polymatroid structure, optimal resource alloctaion and throughput capacities", IEEE Trans. Inform. Theory, vol. 44, pp. 2796-2815, Nov. 1998.

[61] S. V. Hanly, and D. S. C. Tse, "Multiaccess fading channels-part II: delay-limited capacities", IEEE Trans. Inform. Theory, vol. 44, pp. 2816-2831, Nov. 1998.

[62] A. J. Goldsmith, and L. Greenstein, "A measurement-based model for predicting coverage areas of urban microcells," IEEE Trans. Veh. Technol., vol. 117, pp. 10131023, Sep. 1993.

[63] N. B. Mehta, "Impact of user mibility on resource allocation schemes in cellular radio systems," Ph.D Thesis, California Institute of Technology, California, Jan. 2001

[64] A. J. Goldsmith, Wireless Communications. Cambridge University Press: Cambridge, 2005 .

[65] V. Erceg, L. J. Greenstein, S. Y. Tjandra, S. R. Parkoff, A. Gupta, B. Kulic, A. A. Julius, and R. Bianchi, "An empirically based path loss model for wireless channels in suburban environments," IEEE J. Select. Areas Commun., vol. 17, pp. 12051211, Jul. 1999.

[66] M. K. Simon, and M-S. Alouini, Digital Communication over Fading Channel. New York: John Wiley \& Sons, Inc, 2000. 
[67] Q.T. Zhang,"Outage probability in cellular mobile radio due to Nakagami signal and interferers with arbitary parameters," IEEE Trans. Veh. Technol., vol. 45, pp. 364-372, May. 1996.

[68] H. M. Sachs, "A realistic approach to defining the probability of meeting acceptable receiver performance criteria," IEEE Trans. Elect. Magnet. Compat., vol. 13, pp. 3-6, 1971.

[69] L. Li, and A. J. Goldsmith, "Minimum outage probability and optimal power allocation for fading multiple-access channels," in Proc. Int. Symp. Information Theory (ISIT'00), June 2000, p. 75

[70] R. Negi, M. Charikar, and J. Cioffi, "Minimum outage transmission over fading channel with delay constraint," in Proc. IEEE ICC'00, June 2000, pp. 282-286.

[71] R. Knopp, and P. A. Humblet, "Information capacity and power control in singlecell multiuser communications," in Proc. ICC'95, Jun. 1995, pp. 331-335.

[72] R. Frenkiel, B. R. Badrinath, J. Borras, and R. D. Yates, "The infostations challenge: balancing cost and ubiquity in delivering wireless data," IEEE Pers. Commun. Mag., vol. 7, pp. 66-71, Feb. 2000.

[73] S. Ulukus, and L. J. Greenstein, "Throughput maximization in CDMA uplinks using adaptive spreading and power control," in Proc. IEEE 6th Int. Symp. SpreadSpectrum Techniques, Applicayions, Sep. 2000, pp. 565-569.

[74] N. Feng, S.-C. Mau, and N. B. Mandayam, "Pricing and power control for joint network-cenric and user-centric radio resource management," IEEE Trans. Commun., vol. 52, pp. 1547-1557, Sep. 2004.

[75] R. D. Yates, and N. B. Mandayam, "Challenges in low-cost wireless data transmission," IEEE Signal Processing Mag., May. 2000, pp.93-102. 
[76] D. Famolari, N. B. Mandauam, and D. J. Goodman "A new framework for power control in wireless data networks: games, utility and pricing," in Proc. Allerton Conf. Commun., Control, and Computing, Sep. 1998, pp. 546-555.

[77] N. Feng, N. B. Madayam, and D. J. Goodman "Joint power and rate optimization for wireless data services based on utility functions," in Proc. CISS., vol. 1, Mar. 1999, pp. 109-113.

[78] V. Shah, N. B. Madayam, and D. J. Goodman, "Power control for wireless data based on utlity and pricing," in Proc. PIMRC, 1998, pp. 1427-1432.

[79] A. Mass-Collel, M. D. Whinston, and J. R. Green Microeconomic Theory. Oxford Univ. Press: Oxford, U.K., 1995.

[80] D. Fudenberg, and J. Tirole, Game Theroy. MIT Press: Cambridge, MA, 1991.

[81] C. U. Saraydar, N. B. Mandayam, and D. J. Goodman, "Pricing and power control in a multicell wireless data network," IEEE J. Select. Areas Commun., vol. 1, pp. 1883-1892, Oct. 2001.

[82] S. Dey, and J. Evans "Optimal power control in wireless data networks with outage-based utility guarantees," in Proc. of IEEE Conf. Decision and Control, Maui, USA, vol. 1, pp. 570-575, Dec. 2003.

[83] J. G. Proakis, Digital Communications, 2nd ed. New York: McGrawHill, 1989.

[84] S. T. Chung, and A. J. Goldsmith, "Degrees of freedom in adaptive modulation: a unified view," IEEE Trans. Commun., vol. 49, No. 9, pp. 1561-1571, Sep. 2001. 


\section{Appendix A}

\section{A.1 Proof of Probability Identity}

Using the similar procedures as [48], we give a self-contained derivation of an identity that is used to find an expression of outage probability in chapter 3. Let us assume that $z_{1}, \cdots, z_{n}$ are independent exponentially distributed random variables with means $E\left[z_{i}\right]=\frac{1}{\lambda_{i}}$. That is, the pdf of $z_{i}$ is $f_{Z}\left(z_{i}\right)=\lambda_{i} \exp \left(-\lambda_{i} z_{i}\right)$. Therefore, the identity is expressed as,

$$
\operatorname{Prob}\left(z_{1} \leq c+\sum_{i=2}^{n} z_{i}\right)=1-e^{-\lambda_{1} c} \prod_{i=2}^{n}\left(\frac{1}{1+\frac{\lambda_{1}}{\lambda_{i}}}\right)
$$

Proof: We note that

$$
\begin{aligned}
\operatorname{Prob}\left(z_{1}>c+\sum_{i=2}^{n} z_{i}\right) & =\int_{t_{2}=0}^{\infty} \cdots \int_{t_{n}=0}^{\infty} \operatorname{Prob}\left(z_{1}>c+\sum_{i=2}^{n} t_{i}\right) \prod_{i=2}^{n} \lambda_{i} e^{\left(-\lambda_{i} t_{i}\right)} d t_{2} \cdots d t_{n} \\
& =\int_{t_{2}=0}^{\infty} \cdots \int_{t_{n}=0}^{\infty} e^{-\lambda_{1}\left(c+t_{2}+\cdots+t_{n}\right)} \prod_{i=2}^{n} \lambda_{i} e^{\left(-\lambda_{i} t_{i}\right)} d t_{2} \cdots d t_{n} \\
& =e^{-\lambda_{1} c} \prod_{i=2}^{n} \int_{t_{i}=0}^{\infty} \lambda_{i} e^{-\left(\lambda_{1}+\lambda_{i}\right) t_{i}} d t_{i} \\
& =e^{-\lambda_{1} c} \prod_{i=2}^{n} \frac{\lambda_{i}}{\lambda_{1}+\lambda_{i}}
\end{aligned}
$$

Therefore,

$$
\operatorname{Prob}\left(z_{1} \leq c+\sum_{i=2}^{n} z_{i}\right)=1-e^{-\lambda_{1} c} \prod_{i=2}^{n} \frac{\lambda_{i}}{\lambda_{1}+\lambda_{i}}
$$




\section{A.2 Davidon-Fletcher-Powell Algorithm}

The steps for finding minimum value of function $f(\vec{x})$ and its corresponding optimal variable using Davidon-Fletcher-Powell (DFP) algorithm are given below:

Step 1: Set iteration index $k=0$ and input tolerance $\epsilon$ to some value. Initialize optimization variable $\vec{x}^{(0)}$ to some value and direction matrix $\mathbf{S}^{(0)}=\mathbf{I}_{N}$, where $\mathbf{I}_{N}$ is a $N \times N$ identity matrix.

Step 2: Compute gradient vector $\vec{g}^{(0)}$ at initial point $\vec{x}^{(0)}$.

Step 3: Compute DFP direction vector using following relation,

$$
\vec{d}^{(k)}=-\mathbf{S}^{(k)} \vec{g}^{(k)}
$$

Step 4: Using a line search technique (e.g., Fletcher line search), find the value of $\alpha^{(k)}$ (a small constant) that minimizes

$$
f\left(\vec{x}^{(k)}+\alpha^{(k)} \vec{d}^{(k)}\right)
$$

Step 5: Set optimal direction vector $\vec{\delta}^{(k)}=\alpha^{(k)} \vec{d}^{(k)}$ and find updated value of variable

$$
\vec{x}^{(k+1)}=\vec{x}^{(k)}+\vec{\delta}^{(k)}
$$

Step 6: If $\left|\vec{\delta}^{(k)}\right|<\epsilon$, then output

$$
\begin{aligned}
\vec{x}^{*} & =\vec{x}^{(k+1)}, \text { and } \\
f\left(\vec{x}^{*}\right) & =f\left(\vec{x}^{(k+1)}\right)
\end{aligned}
$$

and then stop.

Step 7: Compute gradient vector at updated point $\vec{g}^{(k+1)}$ and set

$$
\vec{\beta}^{(k)}=\vec{g}^{(k+1)}-\vec{g}^{(k)}
$$


Step 8: Compute new direction vector $\vec{S}^{(k+1)}$ using following updating formula:

$$
\vec{S}^{(k+1)}=\vec{S}^{(k)}+\frac{\vec{\delta}^{(k)}\left(\vec{\delta}^{(k)}\right)^{T}}{\left(\vec{\delta}^{(k)}\right)^{T} \vec{\beta}^{(k)}}-\frac{\vec{S}^{(k)} \vec{\beta}^{(k)}\left(\vec{\beta}^{(k)}\right)^{T} \vec{S}^{(k)}}{\left(\vec{\beta}^{(k)}\right)^{T} \vec{S}^{(k)} \vec{\beta}^{(k)}} .
$$

Step 9: Set $k=k+1$ and repeat from Step 2 . 


\section{A.3 Broyden-Fletcher-Goldfarb-Shanno Algorithm}

The steps for finding minimum value of function $f(\vec{x})$ and its corresponding optimal variable using Broyden-Fletcher-Goldfarb-Shanno (BFGS) algorithm are given below:

Step 1: Set iteration index $k=0$ and input tolerance $\epsilon$ to some value. Initialize optimization variable $\vec{x}^{(0)}$ to some value and direction matrix $\mathbf{S}^{(0)}=\mathbf{I}_{N}$, where $\mathbf{I}_{N}$ is a $N \times N$ identity matrix.

Step 2: Compute gradient vector $\vec{g}^{(0)}$ at initial point $\vec{x}^{(0)}$.

Step 3: Compute BFGS direction vector using following relation,

$$
\vec{d}^{(k)}=-\mathbf{S}^{(k)} \vec{g}^{(k)}
$$

Step 4: Using a line search (e.g., Fletcher line search) find the value of $\alpha^{(k)}$ (a small constant) that minimizes the following relation,

$$
f\left(\vec{x}^{(k)}+\alpha^{(k)} \vec{d}^{(k)}\right)
$$

Step 5: Set optimal direction vector $\vec{\delta}^{(k)}=\alpha^{(k)} \vec{d}^{(k)}$ and find updated value of variables

$$
\vec{x}^{(k+1)}=\vec{x}^{(k)}+\vec{\delta}^{(k)}
$$

Step 6: If $\left|\vec{\delta}^{(k)}\right|<\epsilon$, then output

$$
\begin{aligned}
\vec{x}^{*} & =\vec{x}^{(k+1)} \text { and } \\
f\left(\vec{x}^{*}\right) & =f\left(\vec{x}^{(k+1)}\right),
\end{aligned}
$$

and then stop.

Step 7: Compute gradient vector at updated point $\vec{g}^{(k+1)}$ and set

$$
\vec{\beta}^{(k)}=\vec{g}^{(k+1)}-\vec{g}^{(k)}
$$


Step 8: Compute new direction vector $\vec{S}^{(k+1)}$ using following updating formula:

$$
\vec{S}^{(k+1)}=\vec{S}^{(k)}+\left(1+\frac{\left(\vec{\beta}^{(k)}\right)^{T} \vec{S}^{(k)} \vec{\beta}^{(k)}}{\left(\vec{\beta}^{(k)}\right)^{T} \vec{\delta}^{(k)}}\right) \frac{\vec{\delta}^{(k)}\left(\vec{\delta}^{(k)}\right)^{T}}{\left(\vec{\beta}^{(k)}\right)^{T} \vec{\delta}^{(k)}}-\frac{\vec{\delta}^{(k)}\left(\vec{\beta}^{(k)}\right)^{T} \vec{S}^{(k)}+\vec{S}^{(k)} \vec{\beta}^{(k)}\left(\vec{\delta}^{(k)}\right)^{T}}{\left(\vec{\beta}^{(k)}\right)^{T} \vec{\delta}^{(k)}} .
$$

Step 9: Set $k=k+1$ and repeat from Step 2 . 